\title{
Análisis de los factores determinantes de la transparencia en RSC en las empresas españolas cotizadas
}

\author{
Adrian Testera Fuertes, Laura Cabeza García \\ Universidad de León (Spain)
}

atesf@unileon.es, laura.cabeza@unileon.es

Submitted: November 2011

Accepted: August 2012

\section{Resumen}

Objeto: Este trabajo pretende profundizar en los determinantes de la divulgación de información sobre las acciones sociales que las empresas realizan. En particular, se centra en el papel que puede jugar un factor apenas considerado hasta el momento como es la estructura de propiedad.

Diseño/metodología: Con el objetivo anterior, a partir de una muestra de empresas españolas cotizadas en el año 2008 hemos realizado un análisis de regresión lineal para analizar cómo la identidad del principal accionista de la empresa así como otras características empresariales afectan a la transparencia en RSC.

Aportaciones y resultados: En contra de lo esperado, los resultados sugieren que la identidad del principal accionista no afecta a la transparencia informativa en RSC. Por el contrario, son otras características, como el tamaño de la empresa, su nivel de endeudamiento así como su nivel de transparencia en general, las que parecen explicar la transparencia sobre acciones socialmente responsables.

Limitaciones: La existencia de otros indicadores de transparencia en RSC así como el haber realizado el estudio en un único país y en un momento temporal concreto, hace necesario ser cautos sobre los hallazgos obtenidos.

Valor añadido: Un número reducido de estudios ha analizado la influencia de la estructura de propiedad en la transparencia en RSC, y además, de los pocos trabajos existentes, la mayoría se han centrado en el grado de concentración de la propiedad y no han tenido en cuenta la identidad o tipología del primer accionista. 
Palabras clave: Transparencia, RSC, estructura de propiedad, identidad gran accionista.

Códigos JEL: M14, G30

Title: Analysis of the determinants of CSR disclosure in spanish listed companies

\section{Abstract}

Purpose: This paper aims to analyse in depth the determinants of disclosure of corporate social activities. In particular, it is focused on a barely considered factor: ownership structure.

Design/methodology/approach: Using a sample of Spanish listed companies in 2008 we employed a OLS model to analyse how the identity of the largest shareholder as well as other firm characteristics may influence the disclosure of social activities.

Findings and Originality: Contrary to our expectations, the findings suggest that the identity of the main shareholder does not affect the disclosure of social activities. On the contrary, firm size, leverage and general level of disclosure seem to explain the information provided by the firms related to their social activities.

Research Limitations/implications: Our results should be considered with caution because we have used a specific measure of disclosure of social activities and our study is carried out in a specific country and period of time.

Originality/value: A reduced number of studies have analysed the influence of firm ownership structure on the disclosure of social activities, and the existing studies have mainly considered the ownership concentration but not the identity of the largest shareholder.

Keywords: Disclosure, corporate social activities, ownership structure, identity largest shareholder

JEL Codes: M14, G30

\section{Introducción}

En las últimas décadas, la maximización del beneficio económico para los accionistas ha dejado de ser considerado como el único objetivo empresarial pues, a partir de la teoría de los stakeholders (Freeman, 1984) se ha puesto de manifiesto la necesidad de considerar los intereses de todos los grupos que participan, directa o indirectamente, en la actividad 
empresarial. Como resultado, la responsabilidad de las empresas se ha ampliado más allá de la contraída con sus accionistas siendo necesario atender a una amplia gama de agentes interesados en conocer, además de los aspectos económicos y financieros de la compañía, las relaciones de la misma con el medioambiente y con los grupos sociales de referencia (Archel, 2003).

Por ello, es también habitual que las empresas informen sobre sus actividades de responsabilidad social corporativa (en adelante RSC) en su informe anual o en una memoria social independiente. Sin embargo, no existe un modelo uniforme que estandarice los parámetros sobre los que se debe informar o la manera de confeccionarla. Consecuentemente, numerosas organizaciones han desarrollado modelos que sirvan de guía a la hora de elaborar estas memorias, tales como la Internationally Standards Organization (ISO), el World Resources Institute (WRI) o el Global Reporting Initiative (GRI).

La mayor parte de los estudios previos relativos a los factores explicativos de la transparencia o divulgación de información sobre cuestiones sociales y medioambientales se han centrado en determinadas características de la compañía, sobre todo en su tamaño (Archel, 2003; Roberts, 1992), en el sector de actividad (Moneva \& Llena, 2000; Reverte, 2009) y en su nivel de rentabilidad (Ghazali, 2007). Sin embargo, un número reducido de trabajos considera la influencia que la estructura de propiedad de la empresa puede tener sobre la realización de actividades de RSC, y en concreto, sobre la transparencia informativa en ese sentido, a pesar de que, en la definición del nivel de compromiso de la compañía con los distintos stakeholders, los propietarios pueden resultar determinantes. Además, los pocos estudios existentes han tratado de analizar, en su mayoría, el efecto del grado de concentración de la propiedad sobre la transparencia en RSC (Brammer \& Pavelin, 2008; Ghazali, 2007; Reverte, 2009; Roberts, 1992), no habiéndose considerado apenas la influencia de la identidad o tipología del principal accionista de la empresa, con la excepción, hasta donde nuestro conocimiento alcanza, de Ghazali (2007) o Haniffa y Cooke (2005) para Malasia, Ndemanga y Koffi (2009) para Suecia o Kuo et al. (2012) y Zeng et al. (2012) para China.

En este contexto, el objetivo del presente trabajo es analizar la influencia que la identidad del primer gran accionista de la empresa junto con otras variables tradicionalmente ya consideradas (rendimiento empresarial, tamaño, sector de actividad, endeudamiento y grado de transparencia en general) tienen sobre el nivel de divulgación de información social corporativa para una muestra de empresas españolas cotizadas en el Índice General de la Bolsa de Madrid (IGBM) en el año 2008. Para ello, hemos considerado un índice de transparencia en base a las recomendaciones del Global Reporting Iniciative (GRI) así como un posible problema de endogeneidad en el modelo planteado.

Hasta este momento, la evidencia empírica existente para el caso español, aunque no demasiado abundante, ha constatado que determinadas características de la empresa, tales 
como su nivel de rentabilidad (Prado-Lorenzo, García-Sánchez \& Gallego-Álvarez, 2009), su tamaño (Archel, 2003; García-Ayuso \& Larrinaga, 2003; Prado-Lorenzo et al., 2009; Reverte, 2009), su sector de actividad (Archel, 2003; García-Ayuso \& Larrinaga, 2003; Reverte, 2009) o endeudamiento (García-Ayuso \& Larrinaga, 2003), influyen en el nivel de transparencia informativa en cuestiones sociales y medioambientales. La estructura de propiedad y la de gobierno corporativo parecen afectar también al nivel de divulgación de información social. Así, Reverte (2009) concluye que cuanto mayor sea el grado de concentración de la propiedad menor es el nivel de transparencia informativa, mientras que Prado-Lorenzo et al. (2009) encuentran que aquellas empresas con consejos de administración formados mayoritariamente por independientes y con una mayor diversidad son más transparentes en RSC.

Los resultados del análisis realizado en este trabajo ponen de manifiesto que la identidad del primer gran accionista no parece influir de manera significativa sobre la transparencia en RSC. Por el contrario, las empresas de mayor tamaño, con un menor nivel de endeudamiento y con mayor nivel de transparencia en otras cuestiones parecen divulgar más información sobre las acciones de responsabilidad social que realizan.

El resto del artículo se ha estructurado de la siguiente manera. En el segundo apartado se desarrolla la hipótesis a contrastar basándose en la revisión de la literatura relacionada con el tema objeto de estudio. La muestra, las variables utilizadas y la metodología se describen en el tercer apartado. En la cuarta sección se ofrecen los resultados y, finalmente, se presentan las principales conclusiones del trabajo.

\section{Revisión de la literatura y propuesta investigadora}

En contraposición al objetivo único de maximizar los beneficios de las empresas por parte de sus propietarios (Friedman, 1962), de la mano del desarrollo de la teoría de los stakeholders (Agle, Mitchell \& Sonnenfeld, 1999; Freeman, 1984; Mitchell, Agle \& Wood, 1997) ha surgido el debate acerca de la idoneidad de este planteamiento, poniéndose de manifiesto la necesidad estratégica de considerar a todos los grupos de interés relevantes para la actividad empresarial. Así, muchas compañías han tendido a ampliar sus objetivos, no centrándose exclusivamente en su rendimiento económico, sino también en su desempeño social y medioambiental. Este creciente interés por una gestión de la empresa que atiende las necesidades de sus stakeholders ha contribuido a la consolidación de la RSC, es decir, las acciones voluntarias tomadas por las compañías respecto a sus empleados, a la sociedad y al medioambiente, acciones que van más allá de las requeridas legalmente (Barnea \& Rubin, 2010). Por tanto, la RSC implica una nueva filosofía empresarial que trata de buscar la excelencia en las relaciones de la empresa con todos sus grupos de interés a través de la superación de las exigencias legales y de la máxima calidad de sus comportamientos en los ámbitos social y medioambiental. 
En este sentido, las acciones de RSC pueden favorecer un incremento en la competitividad y rentabilidad de la compañía al poder ser instrumentos adecuados para obtener contratos con empresas o gobiernos (Ruhnka \& Boerstler, 1998), facilitar el acceso a nuevos mercados (Wotruba, 1997), diferenciarse de los competidores (Lev, Petrovitis \& Radhakrishnan, 2006), aumentar la reputación (Diller, 1999), y atraer a inversores y consumidores socialmente responsables (Bagnoli \& Watts, 2003). Adicionalmente, las prácticas de responsabilidad con proveedores, clientes y comunidad local, inciden directamente sobre aspectos clave del contexto competitivo de las empresas ya que mejoran la oferta de inputs especializados de alta calidad, potencian una demanda sofisticada y exigente, y mejoran los sectores relacionados y complementarios (Porter \& Kramer, 2002). Además, la RSC puede ayudar a la empresa a aumentar su eficiencia técnica y operativa gracias a la retención del personal con mas talento o a una mejora en el clima laboral (Albinger \& Freeman, 2000; Turban \& Greening, 1997), a la reducción del coste de financiación y el importe de las primas de los seguros (Lenox \& Nash, 2003), o al no incurrir en sanciones sociales (Aguilera, Rupp, Williams \& Ganapathi, 2007; Freeman et al., 2010).

Por otro lado, las empresas, normalmente, también informan sobre las prácticas de RSC que llevan a cabo bien en su informe anual o bien a través de la elaboración de informes específicos. Así, en las últimas décadas, las prácticas divulgativas de la información medioambiental y social han aumentado su volumen y complejidad (Gray, Avad, Power \& Sinclair, 2001), pasando de ser iniciativas nacionales a convertirse en un auténtico modelo de información social corporativa internacional. En este sentido, varias organizaciones no gubernamentales, como por ejemplo, el Global Reporting Iniciative (GRI), han desarrollado recomendaciones sobre cómo informar acerca de las actividades de RSC.

De acuerdo con la teoría de la legitimidad se considera que la actividad de una empresa está determinada por un contrato social mediante el cual ésta decide llevar a cabo acciones socialmente responsables a cambio de la aprobación de sus objetivos y de otras recompensas, lo que en último término garantiza su supervivencia (Brown \& Deegan, 1998; Deegan, 2002; Guthrie \& Parker, 1989). Este contrato, que cumple las expectativas de ambas partes, tiene un carácter dinámico y puede ser modificado o eliminado cuando alguna de ellas no cumple las expectativas de la otra. En este sentido, el contrato será revocado si las acciones de la empresa son percibidas como algo perjudicial para la comunidad, de manera que los consumidores pueden reducir o dejar de comprarle su producto o servicio, o cuando acciones legales condicionen o prohíban la actividad de la empresa al no estar de acuerdo con las expectativas de la sociedad (Deegan \& Rankin, 1996). En consecuencia, la transparencia en RSC puede ser una estrategia de la compañía para generalizar la percepción de que sus acciones son legítimas, es decir, deseables o apropiadas dentro del sistema social de normas, valores o creencias (Perrow, 1970), asegurando en último término su supervivencia y actividad en la sociedad (Gray, Kouhy \& Lavers, 1995; Hooghiemstra, 2000). 
Por su parte, según la teoría de la agencia, que considera a la empresa como un nexo de contratos entre varios agentes económicos que pueden comportarse de manera oportunista (Jensen \& Meckling, 1976), la transparencia en aspectos sociales y medioambientales puede ser útil a la hora de determinar las obligaciones contractuales en lo que a la financiación con deuda se refiere o en los contratos sobre compensaciones a los directivos (Reverte, 2009).

Sin embargo, en línea con la teoría de los stakeholders, en las empresas se tiene en cuenta el impacto esperado por parte de los diferentes grupos de interés de las políticas de RSC. La divulgación de información social y medioambiental es un intento de la compañía por legitimizar sus actuaciones ante un conjunto de usuarios, diferentes a accionistas y acreedores, interesados en su comportamiento (Blackburn, Jarman \& Siltanen, 1994; Moneva \& Llena, 1996; Patten, 1992; Roberts, 1992). De manera más concreta, la transparencia en RSC puede ser una herramienta para manejar las necesidades de información de los stakeholders con más poder para lograr su apoyo y no poner en peligro su supervivencia (Gray, Owen \& Adams, 1996). En este sentido, a diferencia de la teoría de la legitimidad, esta teoría, considerando que los grupos de interés pueden tener distintos puntos de vista sobre cómo la organización tiene que llevar a cabo su actividad, establece que debería haber diferentes contratos sociales que se negociarían con cada uno de los grupos de interés y no un único contrato con la sociedad. Así, según la perspectiva de la teoría de los stakeholders las compañías realizarán actividades de RSC e informarán sobre ellas a sus grupos de interés para ajustarse a las normas y expectativas que éstos tienen sobre la actividad que la empresa debe realizar y para visualizar la asunción de tales compromisos (Castelo \& Lima, 2008; Roberts, 1992). Ello explicaría que la mayor importancia atribuida a la realización de actividades de RSC haya coincidido con un aumento en la incidencia y sofisticación de la divulgación de información sobre estas cuestiones (Campbell, 2004; Gray et al., 1995).

Así pues, en línea con los argumentos anteriores, la transparencia en RSC puede derivar en una mayor legitimidad con todos los grupos de interés así como favorecer un aumento de la imagen y reputación empresarial (Castelo \& Lima, 2008) y satisfacer las necesidades de información de todos aquellos que utilicen estos informes. Por ello, la transparencia sobre las acciones sociales y la realización de dichas actividades pueden ser maneras complementarias para lograr una ventaja competitiva en la empresa y contribuir a una mejora en el rendimiento empresarial vía una mejora en la reputación empresarial.

El hecho de que una empresa divulgue más o menos información sobre las actividades de naturaleza social que realiza parece estar determinado tanto por sus propias características como por las de la industria a la que pertenece, las cuales influyen en el coste y beneficio relativo de la transparencia (Belkaoui \& Karpik, 1989; Cormier, Magnan, \& Van Velthoven 2005; Patten, 2002a, 2002b). En este sentido, un posible factor a considerar a la hora de explicar la transparencia en RSC es la estructura de propiedad de la empresa al influir en las 
decisiones y actuaciones empresariales, dado que no todos los propietarios pueden tener el mismo peso y capacidad para influir en la estrategia de la organización, dentro de la cual se engloban las acciones de RSC, y a que sus objetivos pueden ser distintos (Ortiz de Mandojana, Aragón \& Delgado, 2011).

En aquellas compañías con una estructura de propiedad más concentrada, la RSC, y también su divulgación, puede tener más peso en la toma de decisiones para evitar el coste de ser calificada como una empresa "socialmente irresponsable" (Barnea \& Rubin, 2010). De igual forma, considerando que los grandes accionistas pueden tener un objetivo distinto al rendimiento a corto plazo asociado a sus inversiones (Blair, 1995; Monks \& Minow, 1995), las empresas que cuenten con una propiedad más concentrada dispondrán de un abanico más amplio de posibles inversiones $y$, entre ellas, tendrán especial cabida las de mayor horizonte temporal (Hoopes \& Miller, 2006), como por ejemplo, inversiones en RSC (Johnson \& Greening, 1999; Mahapatra, 1984). Por otro lado, la realización de actividades de naturaleza social puede hacer más fácil la obtención de fondos así como la reducción de costosas sanciones derivadas de decisiones judiciales, de una legislación adversa presente o futura y de posibles represalias de los consumidores (Consolandi, Nascenzi \& Jaiswal-Dale, 2008; Graves \& Waddock, 1994).

Sin embargo, también existen argumentos que abogan por una relación negativa entre la concentración de la propiedad y la RSC o la transparencia en esta materia debido, en primer lugar, a que el principal accionista puede tener un poder excesivo que le permita tomar decisiones encaminadas a la obtención de beneficios privados en detrimento del interés de los accionistas minoritarios (Becht \& Röel, 1999; Faccio, Lang \& Young, 2001; Morck, Wolfenzon \& Yeung, 2005). Además, en las empresas de propiedad dispersa puede existir una mayor demanda de compromiso social y actividades relacionadas con la comunidad, así como una mayor transparencia e información al respecto (Ghazali, 2007) para, de esta manera, reducir problemas de asimetría informativa entre la compañía y sus accionistas (Prencipe, 2004).

La evidencia empírica en este sentido tampoco es concluyente. Así, algunos estudios encuentran una relación positiva entre la concentración de la propiedad y la implantación de prácticas de RSC (Consolandi et al., 2008; Godos-Díez, Fernández-Gago \& Cabeza-García, 2012; Ndemanga \& Koffi, 2009), mientras que otros concluyen lo contrario (Barnea \& Rubin, 2010; Li \& Zhang, 2010; López-Iturriaga \& López-De-Foronda, 2011). De igual forma, los trabajos previos ponen también de manifiesto una influencia negativa de la concentración de la propiedad sobre la divulgación de las actividades sociales y medioambientales realizadas por la empresa (Ghazali, 2007; Reverte, 2009; Roberts, 1992; Ullmann, 1985) o no encuentran una relación significativa (Brammer \& Pavelin, 2008); pero en ninguno se ha constatado una relación positiva.

Sin embargo, a la hora de analizar qué tipo de actividades de RSC llevan a cabo las empresas, en qué medida lo hacen $y$, en consecuencia, también cuál es su grado de transparencia 
informativa al respecto, es necesario considerar que el papel desempeñado por los grandes accionistas no solo depende de su participación en la propiedad, sino también de su identidad o su naturaleza. Así, la dirección o el equipo directivo de una compañía puede tener una percepción distinta sobre la importancia de ser social y ambientalmente responsable en función de las expectativas de los diferentes propietarios en términos del beneficio económico y del desempeño social y ambiental (Ndemanga \& Koffi, 2009). En este sentido, la mayor parte de los estudios previos se han centrado en la influencia que determinados inversores (sobre todo familias e inversores institucionales) pueden tener sobre la realización de actividades de naturaleza social o la transparencia en RSC (Estado o inversor extranjero).

Tal y como se ha comentado, nuestro objetivo es analizar la influencia de la identidad del primer accionista sobre la transparencia en RSC, por lo que a continuación, se comentarán los fundamentos teóricos sobre cómo la tipología del principal propietario de la empresa puede afectar a la divulgación en materia de RSC así como la evidencia empírica existente hasta el momento.

En lo relativo a la empresa familiar, su interés está en un horizonte temporal a largo plazo, en su supervivencia y en su compromiso con una mejora en la popularidad de la empresa (Anderson, Mansi \& Reeb, 2003; McVey \& Draho, 2005). Por ello, cabría esperar que las empresas familiares tiendan a estar más comprometidas con la reputación corporativa, sean más proclives a involucrase en actividades altruistas para reforzar su ego (Schulze, Lubatkin \& Dino, 2003) y a invertir en acciones de RSC (Déniz \& Cabrera, 2005) para mejorar así el interés de todos los stakeholders y reducir cualquier conflicto de intereses. En consecuencia, las familias o inversores individuales pueden perseguir objetivos no de naturaleza económica (Anderson \& Reeb, 2003; Sharma, Chrisman \& Chua, 1997) distintos a los de los inversores anónimos que buscan una alta rentabilidad (La Porta, Lopez-De-Silanes \& Shleifer, 1999; Miller \& Le Breton-Miller, 2006). Los propietarios familiares pueden estar más inclinados a aplicar estrategias ambientales para evitar así ser calificadas como irresponsables ciudadanos corporativos, lo que empañaría el nombre de la familia. De igual forma, puede ser esperable que las familias reporten también más información sobre las acciones de RSC que llevan a cabo como medio para mejorar su reputación e imagen, contar con el apoyo social y, en último término, asegurar su supervivencia. Es decir, a pesar del riesgo asociado a las inversiones en actividades de RSC en lo que se refiere a la obtención segura de un rendimiento financiero en el largo plazo, una familia puede estar dispuesta a asumirlo, a cambio del mantenimiento de su riqueza socio-emocional y de una buena reputación (Gómez-Mejía, Akács Haynes, NúñezNickel, Jacobson \& Moyano-Fuentes, 2007).

En este sentido, Graafland (2002) concluyó que las empresas familiares holandesas, sobre todo las de gran tamaño, muestran un mayor interés por la RSC que las empresas no familiares. Por su parte, en el trabajo de Berrone, Cruz, Gómez-Mejía y Larraza-Kintana (2010) 
para una muestra de compañías americanas se constata que, al margen de cualquier consideración política o económica, cuando la preservación de la riqueza socio-emocional de la empresa tiene prioridad, como ocurre con los propietarios familiares, es más probable que la compañía participe profundamente en el cumplimiento social.

Por el contrario, también es posible argumentar que una familia que tenga invertida en una empresa una parte importante de su riqueza esté más interesada en el retorno de sus inversiones, es decir, en recibir una ganancia económica que compense el riesgo de la inversión realizada y que garantice la viabilidad de la empresa para las futuras generaciones familiares $y$, en consecuencia, que no se preocupe tanto por las cuestiones ambientales y sociales ni por su transparencia. Así, Ndemanga y Koffi (2009) encontraron que aquellas compañías en las cuales el mayor porcentaje de participación y derecho de voto está en manos de una familia son menos transparentes en sus prácticas de RSC. De manera similar, Burak y Morante (2007) probaron que existe un vínculo entre la estructura de gobierno y la RSC, al demostrar que las empresas cuyos propietarios son una familia están más centradas en la obtención de un rendimiento asociado a sus inversiones $\mathrm{y}$, por tanto, tratarán de reducir su gasto en actividades de responsabilidad social. El trabajo de López-Iturriaga y López-deForonda (2011) para una muestra de empresas europeas sugiere una relación negativa entre la presencia de una familia o individuos en el capital de la compañía como principal accionista y la realización de acciones de RSC.

Por su parte, Déniz y Cabrera (2005), para el caso español, ponen de manifiesto que la mayoría de las empresas familiares, en línea con el enfoque clásico hacia la RSC, no piensan que ésta sea una fuente de ventaja competitiva pero sí que ellas disponen de los recursos y capacidades necesarios para realizar acciones sociales. Se trataría de un perfil filantrópico respecto a la RSC, ya que consideran que deben mantener una amplia relación con la sociedad a pesar de que es percibida como un coste. De igual forma, otro grupo de empresas considera que ni la realización de actividades sociales es una fuente de ventaja competitiva ni que ellas tengan los recursos necesarios para hacer este tipo de actividades. Por último, el grupo más reducido de compañías, aun reconociendo que ellas no tienen los recursos y capacidades necesarios, ven a la RSC como fuente de ventaja competitiva.

Los inversores institucionales, tales como fondos de pensiones, banca de inversión o compañías aseguradoras, pueden preocuparse por las acciones de RSC al ser la reputación social de la compañía un indicador de la competencia en la gestión, o bien por la existencia de otros motivos morales, en función de lo que es justo e imparcial, como consecuencia de las leyes sociales. Además, este tipo de inversores pueden realizar más RSC para mitigar el riesgo de una acción regulatoria adversa o mayores costes de cumplimiento etc. (Neubaum \& Zhara, 2006; Spicer, 1978). Por el contrario, no se descarta que los inversores institucionales reduzcan sus gastos o su inversión en RSC dado el horizonte temporal a largo plazo de este 
tipo de inversiones y el resultado incierto asociado a ellas (Coffey \& Fryxell, 1991). La evidencia empírica en este sentido no es concluyente. En algunos estudios se ha encontrado una relación positiva entre la presencia de un inversor institucional y la realización de RSC. Es el caso, por ejemplo, de Ndemanga y Koffi (2009) para Suecia o de Graves y Waddock (1994), Johnson y Greening (1999) o Neubaum y Zahra (2006) para EEUU. Por el contrario, también para muestras de empresas estadounidenses Coffey y Fryxell (1991) o Arora y Dhawadkar (2011) encuentran una influencia negativa de la propiedad institucional sobre los niveles de RSC.

Una posible explicación para una relación tanto positiva como negativa entre un inversor institucional y la realización de prácticas de RSC puede ser el horizonte temporal del inversor. Inversores institucionales que se pueden considerar transitorios debido a su orientación a corto plazo (Bushee, 1998, 2001; Porter, 1992) pueden estar más interesados en ganancias trimestrales y en reducir cualquier tipo de actividad de RSC (Neubaum \& Zahra, 2006), mientras que aquellos inversores institucionales con un horizonte temporal a largo plazo estarán más comprometidos con el desempeño social de la empresa al considerar que puede derivar en un impacto financiero positivo a lo largo del tiempo. Así, los resultados de Johnson y Greening (1999) y de Harjoto y Jo (2008) para Estados Unidos y de Aguilera, Williams, Conley, y Rupp (2006) para Reino Unido y Estados Unidos sugieren que aquellos inversores con una orientación a largo plazo, como por ejemplo, los fondos de pensiones y las compañías de seguros, es más probable que tengan en cuenta consideraciones sociales y medioambientales a la hora de tomar sus decisiones de inversión.

El Estado, por su parte, puede tener un fuerte interés en promover actividades de RSC como complemento a sus programas ambientales y sociales y servir así a los intereses nacionales en el largo plazo (Mazurkiewicz, 2006). Las empresas participadas por el Estado pueden involucrarse más en actividades de RSC y ser más transparentes para legitimizar su existencia (Ghazali, 2007). Elevados niveles de propiedad estatal crean incentivos a los CEOs (Chief Executive Officer) para lograr objetivos no financieros relacionados con la política del gobierno (por ejemplo, desarrollo de infraestructuras o mantenimiento del empleo), y por tanto, estos objetivos políticos o sociales ejercen presión sobre la empresa para realizar RSC (See, 2009). En este sentido, Roberts (1992) demuestra que las compañías que se enfrentan a un alto nivel de exposición política son más propensas a dar información sobre sus actividades de responsabilidad social. De igual forma, el trabajo de Ndemanga y Koffi (2009) para Suecia, el de Ghazali (2007) para Malasia, y el de Eng y Mak (2003) para Singapur, ponen de manifiesto que en aquellas compañías donde hay una participación significativa del Estado en el capital de las empresas éstas serán más transparentes sobre las actividades sociales que realizan.

Finalmente, la presencia de un inversor extranjero como principal accionista de la compañía también se ha relacionado con la transparencia en actividades de RSC (Ghazali, 2007). Sus 
actuaciones son más visibles para la opinión pública y para el gobierno del país al que pertenece dicha empresa, por lo que un mayor nivel de transparencia puede reducir cualquier posible crítica sobre la política de actuación de ese inversor extranjero, como por ejemplo, el hecho de estar centrado únicamente en los recursos económicos de ese país. Además, con el fin de competir más eficazmente en el mercado de capitales este tipo de empresas pueden proporcionar voluntariamente más información social (Huafang \& Jianguo, 2007). En último lugar, teniendo en cuenta que el contenido y la extensión de la información social y medioambiental divulgada dependen del país en que se elabore (Gray et al., 1995) y de su cultura medioambiental (Guthrie \& Parker, 1990), la participación de un inversor extranjero en el capital puede condicionar la difusión de información en materia de RSC.

Así, para el caso de Malasia y Singapur, Andrew, Gul, Guthrie \& Teoh (1983) encuentran una relación positiva entre la presencia de grandes inversores extranjeros en el capital de las empresas y la divulgación en las memorias anuales sobre las actividades sociales, al igual que Gamble, Hsu, Kackson \& Tollerson (1996) para una muestra de empresas de 27 países. De manera similar, para el caso español, Moneva y Llena (2000), utilizando una muestra de 70 grandes compañías, cotizadas y no cotizadas, en el período 1992-1994, encontraron que las empresas con una matriz extranjera divulgan más información, en especial, en lo que se refiere a los logros medioambientales o a los gastos e inversiones en materia medioambiental. Por el contrario, los resultados de Archel (2003) para una muestra de compañías con actividad en España entre 1994-1998, no muestran diferencias significativas entre el contenido de los informes anuales elaborados por las empresas de capital español y las que tienen presencia mayoritaria de capital extranjero.

De acuerdo con los fundamentos teóricos anteriores y la evidencia empírica proponemos el contraste de la siguiente hipótesis:

Hipótesis: La identidad del principal accionista de la empresa influye en el nivel de transparencia informativa en actividades de RSC.

Sin embargo, además de la estructura de propiedad, y en concreto, de la tipología de los grandes accionistas, otras variables relativas a la empresa deben ser tenidas en cuenta a la hora de explicar la divulgación sobre las actividades de naturaleza social que éstas realizan. A continuación, se hace referencia a algunas de las principales variables de control más frecuentemente consideradas: rendimiento o performance empresarial, tamaño, sector, endeudamiento.

En lo que se refiere a la influencia del rendimiento empresarial sobre la transparencia en RSC, ésta puede ser positiva o negativa. Así, aunque las empresas pueden desear el seguimiento continuo de las reglas normativas de una buena ciudadanía corporativa, su comportamiento real al respecto va a depender de la disponibilidad de recursos. De este modo, disponer de un mejor resultado económico implicaría una mayor probabilidad de poseer excedentes, los cuales 
ofrecerían a las empresas la oportunidad de invertir más en cualquiera de las dimensiones que abarca la RSC (Preston \& O'Bannon, 1997), y también, por consiguiente podrían suministrar más información sobre las actividades de RSC que realizan (Carmona \& Carrasco, 1988; Cowen, Ferreri \& Parker, 1987; Ismail \& Chandler, 2005; Roberts, 1992). La transparencia es una manera de enviar una señal al mercado sobre su buen rendimiento (Akerlof, 1970). Además, un directivo que sabe cómo hacer a una empresa rentable también debería tener el conocimiento necesario y entender la responsabilidad social, derivando así en una mayor transparencia social y medioambiental (Belkaoui \& Karpik, 1989). Los directivos de empresas rentables también pueden tener un mayor interés en dar más información en beneficio de su propia compensación y de su posición dentro de la compañía (Giner, 1997) o puede ser una manera de trasmitir la idea, sobre todo a aquellos stakeholders más preocupados por el medioambiente, de que se puede obtener un beneficio económico positivo sin ir en detrimento del medio ambiente. Así, varios trabajos empíricos han encontrado soporte para una influencia positiva de los resultados empresariales en la realización de acciones socialmente responsables (Brown \& Perry, 1994; McGuire, Sundgren \& Schneeweis, 1988; Orlitzky, Schmidt \& Rynes, 2003; Preston, Sapienza \& Millar, 1991; Wu, 2006) así como en el nivel de transparencia informativa (Balabanis, Phillips \& Lyall, 1998; Prado-Lorenzo et al., 2009; Roberts, 1992; Singh \& Ahuja, 1983).

Por otro lado, una relación negativa entre la rentabilidad empresarial y la transparencia en RSC puede estar justificada porque la inversión en este tipo de actividades deriva en costes adicionales (Balabanis et al., 1998) o por un comportamiento oportunista de los directivos en el contexto de una estructura de compensación ejecutiva vinculada a los beneficios generados a corto plazo. Por el contrario, si el desempeño financiero es bajo, los directivos podrían intentar justificar estos malos resultados embarcándose en programas sociales llamativos y siendo más transparentes, para convencer a los accionistas u otros inversores que las inversiones que la empresa está realizando en el momento actual, por ejemplo sobre medioambiente, tendrán un efecto positivo sobre el rendimiento o posición competitiva en el largo plazo, o bien por ser una manera de distraer la atención sobre los resultados financieros de la empresa.

Otra variable que puede influir en el nivel de transparencia en RSC es el tamaño de la compañía. Tradicionalmente, el tamaño empresarial se ha asociado de forma positiva con el desempeño social (McWilliams \& Siegel, 2000; Waddock \& Graves, 1997) debido a que, a medida que las empresas crecen, concentran mayor atención por parte de los stakeholders y necesitan responder más eficazmente a sus demandas (Burke, Logsdon, Mitchell, Reiner \& Vogel, 1986; Hillman \& Keim, 2001). Las compañías grandes son más visibles para el público (Watts \& Zimmerman, 1986) y para los grupos políticos (Dowling \& Pfeffer 1975), tienen más poder de mercado y generan más noticia. Por lo tanto, son más propensas a ser objeto del resentimiento público, de la hostilidad de los consumidores, de las exigencias laborales de los 
empleados y de la atención de los cuerpos reguladores del gobierno (Hackston \& Milne, 1996; Knox, Maklan \& French, 2006). En consecuencia, la mayor exposición a la opinión pública, el disponer de más recursos y para evitar las regulaciones de los entes públicos y reducir costes políticos (Adams, Hill \& Roberts, 1998; Clarke \& Gibson-Sweet, 1999; Gray et al., 1995; Ness \& Mirza, 1991), puede explicar que la divulgación voluntaria de sus actividades de RSC sea mayor en las empresas de más tamaño.

Así, diversos estudios empíricos han encontrado una relación positiva y significativa entre el tamaño empresarial y la realización de actividades de RSC (Chih, Chih \& Chen, 2000; Patten, 1991; Pava \& Krausz, 1996; Stanwick \& Stanwick, 1998) así como con la transparencia o divulgación de las acciones de naturaleza social (Adams et al., 1998; Archel, 2003; Brammer \& Pavelin, 2006; Cullen \& Christopher, 2002; Eng \& Mak, 2003; Ghazali, 2007; Hamid, 2004; Haniffa \& Cooke, 2005; Hossain, Perera \& Rahman, 1995; Neu, Warsmhe \& Pedwell, 1998; Patten, 1991). Sin embargo, en otros estudios no se concluye una relación significativa ni entre el tamaño de la compañía y las prácticas de RSC (McGuire et al., 1988) ni con su divulgación (Roberts, 1992).

Con independencia del rendimiento y del tamaño empresarial, la literatura también recoge el efecto que el sector de actividad puede tener en la realización de RSC y en su transparencia. Así, las diferencias derivadas del sector al que pertenece la empresa, por ejemplo, en términos de requerimientos de capital, intensidad de mano de obra, potencial de generación de residuos, etc. pueden influir también en el desarrollo efectivo de prácticas de RSC (Graves \& Waddock, 1994; Ndemanga \& Kofi, 2009; Ullman et al., 1985). Mientras que las empresas industriales están obligadas por las leyes y por los grupos de presión a introducir en su proceso productivo aspectos ambientales y sociales o son sometidas a auditorías ambientales, los minoristas y otras compañías del sector servicios se enfrentan a una menor presión en este sentido.

Además, la industria no solo parece explicar el contenido sino también el alcance de la divulgación o transparencia en contenido social y ambiental (Adams et al., 1998; Cowen et al., 1987; Gray et al., 1995). Las empresas que pertenecen a industrias cuyo proceso de producción puede tener una influencia negativa en el medio ambiente, informan y divulgan mucha más información que las empresas de otras industrias (Reverte, 2009). En general, las compañías de la minería, el petróleo y las industrias químicas hacen especial hincapié en la información sobre cuestiones ambientales, de salud y seguridad (Clarke \& Gibson-Sweet, 1999; Jenkins \& Yakovleva, 2006; Line, Hawley \& Krut, 2002; Ness \& Mirza, 1991), mientras que las industrias del sector financiero y de servicios, en general, parecen inclinarse por un informe con más cuestiones sociales y obras filantrópicas (Clarke \& Gibson-Sweet, 1999; Line et al., 2002). 
En este sentido, Ndemanga y Koffi (2009) demuestran que existe vínculo entre la transparencia sobre las prácticas medioambientales de RSC y ciertos tipos de industria, como maquinaria, petroleras, de gas y alcohol. De manera similar, de acuerdo con los resultados de Roberts (1992) y de Reverte (2009), el sector industrial al que pertenece la compañía es una de las variables que mejor explica las variaciones en los niveles de transparencia en RSC, al ser las compañías más sensibles a impactos medioambientales las más transparentes. Por su parte, Brammer y Pavelin (2008) para Reino Unido encuentran también que la calidad de la transparencia informativa es mayor en sectores con una mayor preocupación medioambiental y Prado-Lorenzo et al. (2009) identifican que las empresas españolas cotizadas que pertenecen al sector del petróleo y la energía son las que divulgan más información social.

Finalmente, otra variable que puede influir en la transparencia en RSC es el nivel de endeudamiento de la empresa. Dentro del contexto de la teoría de la agencia, Jensen y Meckling (1976) afirmaron que las empresas con mayor nivel de endeudamiento ofrecen voluntariamente información con el objetivo de reducir sus costes de agencia y, en consecuencia, su coste de capital. En este sentido, el trabajo de García-Ayuso y Larrinaga (2003) para una muestra de empresas españolas cotizadas en el período 1991-1995, concluye que aquellas que proporcionan más información medioambiental en sus informes anuales tienen un mayor nivel de endeudamiento. Por otra parte, un bajo grado de endeudamiento asegura que los acreedores ejercerán menos presión a los directivos de las empresas a cerca de actividades de RSC y su divulgación, dado que éstas están únicamente ligadas de manera indirecta al éxito financiero de la compañía (Brammer \& Pavelin, 2008). El trabajo de Castelo y Lima (2008) para Portugal y Eng y Mak (2003) confirman la idea de que las empresas con un menor nivel de endeudamiento son más transparentes en materia de RSC. Sin embargo, ni los resultados de Reverte (2009) ni los de Brammer y Pavelin (2008) muestran que el endeudamiento explique las diferencias en las prácticas de RSC entre las empresas.

\section{Muestra, variables y metodología}

\section{Muestra}

La base de datos del estudio está compuesta por el conjunto de empresas españolas cotizadas en la Bolsa de Madrid a 31 de diciembre del año 2008, un total de 133 empresas. De esta base de datos hemos excluido las empresas financieras, de seguros y de servicios de inversión por sus especiales características, sobre todo desde el punto de vista contable (22 compañías). De igual forma, con el fin de evitar valores missing en nuestras estimaciones tampoco hemos considerado aquellos casos para los que no disponíamos de información relativa a alguna de las variables utilizadas ( 1 compañía) y aquellas empresas que presentaban una estructura de propiedad dispersa (1 compañía). Tras aplicar estos filtros, la muestra final se compone de 109 empresas españolas cotizadas, que representa aproximadamente el 98 por ciento del total de las empresas no financieras, de seguros o de servicios de inversión. 
La información relativa a la estructura de propiedad la obtuvimos principalmente de los Informes Anuales de Gobierno Corporativo depositados en la Comisión Nacional del Mercado de Valores (CNMV). Además, utilizamos la Bolsa de Madrid, la CNMV y la base de datos SABI (Sociedad de Análisis de Balances Ibéricos) para recopilar la información financiera y sobre el sector de actividad de las empresas así como el Observatorio de la Fundación de Estudios Financieros (FEF) para construir una de las variables de control consideradas -transparencia informativa de la empresa, tal y como comentaremos más adelante-.

\section{Medida de variables}

Variable dependiente. Nuestra variable dependiente es un indicador de la transparencia informativa en RSC. El punto de partida fue identificar dentro de la muestra aquellas empresas que publicaron un informe de responsabilidad social o sostenibilidad bajo las directrices del Global Reporting Iniciative para el ejercicio 2008, un total de 37 empresas (34 por ciento de la muestra). El GRI es una organización compuesta por miles de expertos a lo largo del mundo cuyo objetivo es desarrollar un marco de normas de alcance mundial con las pautas para mejorar la producción y la comprensión de información transparente, fiable y comparable de informes de sostenibilidad. Así, su objetivo principal es la divulgación de información social, medioambiental y económica.

Siguiendo las recomendaciones establecidas por el GRI hemos medido la transparencia informativa que ofrecen las empresas en cuatro índices o dimensiones (Anexo 1). La primera dimensión, Perfil, se compone de 42 ítems o variables sobre los cuales las empresas dan información sobre su estrategia social y medioambiental, de los detalles de su perfil y de la participación y los compromisos del gobierno corporativo en la organización.

En segundo lugar, la dimensión Económica de la sostenibilidad se refiere a los impactos de la organización sobre las condiciones económicas de sus stakeholders así como en los sistemas económicos en que se desenvuelve (locales, nacionales $\mathrm{y}$, en su caso, internacionales) (9 variables).

En tercer lugar, la dimensión Ambiental de la sostenibilidad se ocupa de los impactos que la empresa puede ocasionar sobre la vida de los sistemas naturales, incluidos los ecosistemas, la tierra, el aire y el agua. Las 30 variables ambientales de esta dimensión están relacionadas con las materias primas procedentes de la naturaleza, los residuos enviados al medioambiente, el compromiso de la organización con la biodiversidad o el cumplimiento de las normas ambientales, entre otras.

Por último, la dimensión Social se divide, a su vez, en otras 4 subcategorías (45 variables). La primera de ellas informa si las prácticas laborales se ajustan a la Declaración de Principios y Derechos Fundamentales del Trabajo. La siguiente subcategoría muestra el respeto de la organización hacia los Derechos Humanos, como por ejemplo, no discriminación, igualdad de género, libertad de asociación, trabajo infantil, mano de obra forzada o los derechos indígenas. 
En tercer lugar, se considera el impacto que la compañía puede tener en la comunidad local en la que opera, cómo gestiona los posibles riesgos que pueden surgir de las interacciones con otras instituciones sociales, en la información sobre los peligros asociados a la corrupción y a los sobornos, a la indebida influencia en la política pública y en lo relativo a prácticas monopolísticas. Finalmente, la subcategoría sobre la Responsabilidad sobre los Productos aborda los aspectos de la cobertura de información en los productos y servicios de una organización y que afectan directamente a sus clientes, tales como el etiquetado, la privacidad del cliente y su satisfacción, posibles riesgos para la salud del consumo de ciertos productos, etc.

Además de las cuatro dimensiones anteriores, se han considerado otras cuatro variables vinculadas a la transparencia en RSC de acuerdo también con otras recomendaciones del GRI sobre cuestiones más generales relativas a la memoria de sostenibilidad que las empresas elaboran. De esta forma, se analiza si la empresa ofrece información acerca del proceso de elaboración de dicha memoria; si en ella se incluye un informe de conclusiones de un Comité de Expertos independiente a la empresa que les ayude a impulsar la transparencia de la información divulgada; si incluye un informe que justifique su verificación por una entidad independiente a la propia empresa que revise la información relativa a las prácticas ambientales, de seguridad y sociales contenida en la memoria de sostenibilidad; y si hace mención a las perspectivas o retos futuros que pretende realizar en términos de mejora social o medioambiental.

Para cada una de las compañías se fue comprobando sobre cuál de las 130 variables o ítems informaba en su informe de sostenibilidad o de RSC. En concreto, se definieron variables dummies que toman valor 1 si la empresa proporciona información sobre cada uno de los ítems y cero en caso contrario, y para cada dimensión (Perfil, Económica, Ambiental y Social) se ha construido un índice que representa el grado de información o la puntuación de la empresa sobre el total de ítems considerados en cada una de estas cuatro dimensiones. En último término, hemos construido un índice global de transparencia que engloba los índices de las cuatro dimensiones y las cuatro variables adicionales que se han considerado de acuerdo con las directrices del GRI (TRANSPRSC). Esta metodología, que enfatiza amplitud -que no extensión- de la información divulgada en relación a los elementos seleccionados ha sido utilizada en estudios previos (Archel, 2003; Carmona \& Carrasco, 1988; Patten, 1991, 1992; Wallace, Naser \& Mora, 1994).

De los cuatro subíndices que componen nuestro índice final de transparencia, las empresas y con independencia de quién sea su principal accionista, parecen informar en mayor medida sobre su Perfil de empresa (valor medio de 0,986), seguido del apartado relativo al Desarrollo Económico (valor medio de 0,895), del Desarrollo Medioambiental (valor medio de 0,812) y en menor medida sobre los ítems asociados al Desarrollo Social (valor medio de 0,743 ). De igual 
forma, dentro de este último subíndice las empresas parecen ser más transparentes en lo que se refiere a prácticas laborales y éticas y a la responsabilidad de productos (valor medio de 0,827 y 0,757 , respectivamente) y menos sobre los derechos humanos y la sociedad en general (valor medio de 0,676 y 0,678, respectivamente). Esta distribución, por importancia en lo que se refiere a la información proporcionada en los cuatro sub-índices de la dimensión social, es la que se da también en el caso de las empresas cuyo principal accionista es una familia, una entidad no financiera o financiera. Por el contrario, en otras tipologías la distribución varía en algunos de los casos.

Variables independientes. Variables categóricas relativas a la identidad o tipología del principal accionista de la empresa son las principales variables explicativas en nuestro estudio. En concreto, de manera alternativa hemos definido variables que toman valor 1 si el primer accionista de la empresa es una familia (FAM), un inversor institucional (por ejemplo, fondos de inversiones o sociedades de cartera) (IINS), el Estado (ESTADO), una empresa extranjera (EXTRAN), una entidad no financiera (NFINAN) o una empresa financiera (bancos o cajas de ahorros) (FINAN) y 0 en caso contrario. Ha sido necesaria la consideración de dos tipologías no consideradas en la literatura previa, empresa no financiera y financiera, debido a que en España, en un porcentaje significativo de los casos este tipo de inversores son los principales accionistas de las empresas.

Considerando un posible problema de endogeneidad entre la estructura de propiedad y la transparencia informativa en RSC, y en especial, entre algunas de las variables de control (por ejemplo, la rentabilidad empresarial) y la transparencia, las variables relativas a la identidad del primer gran accionista así como las variables de control, a las que se hará mención más adelante, se corresponden con el año 2007. De manera similar, Roberts (1992) en su trabajo y con un objetivo similar al nuestro, también incluyó las variables explicativas retardadas un año al considerar la naturaleza dinámica del planning estratégico o el interés de la teoría de los stakeholders sobre el cumplimiento en los objetivos a largo plazo de los grupos de interés.

Variables de control. Dado que las acciones de transparencia informativa en responsabilidad social pueden venir condicionadas por diversas características propias de la empresa y del sector al que ésta pertenece, se han incluido una serie de variables de control referidas al año 2007. En primer lugar, se consideró un indicador del rendimiento de la empresa, la rentabilidad financiera, definido como el cociente entre el resultado del ejercicio y los fondos propios (ROE) (Li \& Zhang, 2010; Roberts, 1992). Aunque se pueden utilizar tanto indicadores contables como de mercado, teniendo en cuenta que estos últimos están basados en la opinión de los inversores sobre el rendimiento de la empresa, no considerando así a otros grupos de interés importantes, hemos preferido la utilización de medidas contables.

En segundo lugar, también se tuvo en cuenta el tamaño de la empresa medido por el logaritmo del total de activo (Brammer \& Pavelin, 2008; Castelo \& Lima, 2008; De Villiers, Naiker \& 
Staden, 2011), que es una variable relevante pues se ha asociado tradicionalmente de forma positiva con el desempeño social y la transparencia en esta materia.

En tercer lugar, hemos incluido el sector de actividad al que pertenece la compañía -medido como una variable de tipo dummy que toma valor 1 si la empresa pertenece a sectores "más sensibles" desde el punto de vista medioambiental (minería, petróleo, gas, productos químicos, papel, siderurgia y otros metales, electricidad, distribución de gas y agua) y 0 en caso contrario (De Villiers et al., 2011; Reverte, 2009) al ser necesario controlar las diferencias derivadas por la pertenencia a un determinado sector de actividad.

Se ha considerado también como variable de control el nivel de endeudamiento de la compañía medido como el cociente entre los fondos ajenos (deuda a corto plazo y a largo plazo) y el activo total (Castelo \& Lima, 2008; Eng \& Mak, 2003; Reverte, 2009).

Finalmente, se ha incorporado en el modelo objeto de estudio una variable de control relativa a la tendencia de la empresa a la transparencia informativa en general (TRANSP) al ser esperable que las empresas con mayores índices de transparencia y comunicación sean también las que difundan en mayor medida las acciones de RSC que realicen. En concreto, hemos utilizado el Rating de Buen Gobierno y Transparencia Informativa elaborado por la Fundación de Estudios Financieros (FEF) para las empresas españolas cotizadas y que analiza la transparencia informativa de la empresa en su informe anual y de gestión (estados financieros e información del grupo, información sobre sus activos y pasivos, informe de gestión y otra información) así como sus prácticas de gobierno corporativo. Así, se ha definido como una variable que toma valores crecientes de 0 a 3 cuanto mayor fuera la puntuación de la empresa en función de las 4 categorías del rating de la $\operatorname{FEF}(A, B, C, D)$.

\section{Metodología}

El modelo econométrico que se emplea para el contraste de la hipótesis propuesta está determinado por el tipo de variable que se pretende explicar. Nuestra variable dependiente "Transparencia en RSC" tiene un carácter continuo que toma valor cero en un porcentaje significativo de las observaciones, es decir, en aquellos casos donde las empresas no elaboran un informe de acuerdo con las directrices del GRI. En consecuencia, al tratarse de una variable observable solo para una parte de la muestra se podría estar cometiendo un sesgo de selección muestral. Por ello, a priori la técnica estadística mas adecuada sería el método de estimación en dos etapas de Heckman (1979) que supone, por un lado, la estimación de la ecuación de selección a partir de un modelo probit respecto a la decisión de elaborar un informe de transparencia en RSC siguiendo las directrices del GRI, y por otro lado, estimar la ecuación de regresión corregida (determinantes del índice de transparencia elaborado) gracias a la incorporación de la inversa del ratio de mills, es decir, la probabilidad de estar en la muestra sobre la probabilidad de no estar en ella) mediante MCO. Sin embargo, en todas las estimaciones realizadas el coeficiente que acompaña a la inversa del ratio de mills no resultó 
significativo, de manera que no parece existir sesgo de selección siendo más correcto en ese caso interpretar los coeficientes de una estimación realizada por MCO al ser el mejor estimador lineal insesgado (MELI).

De manera más concreta, el modelo de regresión lineal que se pretende estimar, a fin de determinar los factores explicativos de la transparencia en RSC de las empresas españolas cotizadas es el siguiente:

$\operatorname{TRANPSRSC}_{i t}=a_{0}+\beta_{1}$ TIPOLOGLA $_{i, t-1}+\beta_{2}$ ROE $_{i, t-1}+\beta_{3}$ TAMAÑO $_{i, t-1}+\beta_{4}$ SECTOR $_{i, t-1}+\beta_{5}$ END $_{i, t-1}+\beta_{6}$ TRANSP $_{i, t-1}+\varepsilon_{i}$

donde TRANSRSC es el índice de transparencia en RSC; TIPOLOGÍA hace referencia a todas las variables dummies relativas a la identidad del primer gran accionista (familia, inversor institucional, Estado, empresa extranjera, entidad no financiera y financiera, alternativamente); ROE es la rentabilidad financiera de la empresa; TAMAÑO es el logaritmo del activo total de la empresa; SECTOR toma valor 1 para aquellos sectores "más sensibles" a impactos medioambientales; END es el cociente entre los fondos ajenos y el activo total de la empresa; TRANSP es el rating de transparencia en general y de gobierno corporativo de la empresa; $\varepsilon$ i es el término de error.

\section{Resultados}

La Tabla 1 muestra los principales estadísticos descriptivos de las variables utilizadas en el estudio, mientras que la Tabla 2 recoge las correlaciones bivariadas entre ellas. Aunque algunas de las variables muestran una correlación estadísticamente significativa, siguiendo la regla empírica de Kleinbaum et al. (1998) el examen de los factores de inflación de la varianza (FIV) indicó que no había evidencia de multicolinealidad pues ningún FIV fue superior a 10.

\begin{tabular}{|c|c|c|c|c|c|c|}
\hline Variables & $\mathbf{N}$ & Media & Mediana & Máximo & Mínimo & $\begin{array}{l}\text { Desv. } \\
\text { típica }\end{array}$ \\
\hline TRANSPRSC & 109 & 0,285 & 0 & 0,961 & 0 & 0,407 \\
\hline ROE & 109 & 0,093 & 0,145 & 1,233 & $-1,854$ & 0,338 \\
\hline TAMAÑO & 109 & 6.772 .179 & 947.793 & $1,06 \mathrm{E}+08$ & 36.019 & $1,59 E+07$ \\
\hline END & 109 & 0,611 & 0,641 & 0,967 & 0,085 & 0,186 \\
\hline TRANSP & 109 & 2,311 & 2 & 3 & 0 & 0,662 \\
\hline \multicolumn{3}{|c|}{ Otras variables explicativas } & \multicolumn{4}{|c|}{ Porcentaje / (número de observaciones $=1$ ) } \\
\hline FAM & & & $\begin{array}{c}46,79 \% \\
(51)\end{array}$ & & & \\
\hline IINS & & & $\begin{array}{c}3,67 \% \\
(4)\end{array}$ & & & \\
\hline ESTADO & & & $\begin{array}{c}0,92 \% \\
(1)\end{array}$ & & & \\
\hline EXTRAN & & & $\begin{array}{c}4,59 \% \\
(5)\end{array}$ & & & \\
\hline
\end{tabular}




\begin{tabular}{|l|l|l|c|l|l|l|}
\hline \multicolumn{1}{|c|}{ Variables } & N & Media & Mediana & Máximo & Mínimo & $\begin{array}{c}\text { Desv. } \\
\text { típica }\end{array}$ \\
\hline NFINAN & & & $\begin{array}{c}38,53 \% \\
(42)\end{array}$ & & & \\
\hline FINAN & & & $\begin{array}{c}5,50 \% \\
(6)\end{array}$ & & & \\
\hline SECTOR & & & $\begin{array}{c}32,11 \% \\
(35)\end{array}$ & & & \\
\hline
\end{tabular}

Tabla 1. Estadísticos descriptivos

\begin{tabular}{|c|c|c|c|c|c|c|c|c|c|c|c|}
\hline Variables & 1 & 2 & 3 & 4 & 5 & 6 & 7 & 8 & 9 & 10 & 11 \\
\hline 1TRANPSRSC & 1 & & & & & & & & & & \\
\hline 2FAM & $\begin{array}{c}-0,234 * * \\
(0,014)\end{array}$ & 1 & & & & & & & & & \\
\hline 3IINS & $\begin{array}{l}-0,050 \\
(0,604)\end{array}$ & $\begin{array}{c}-0,183^{*} \\
(0,056)\end{array}$ & 1 & & & & & & & & \\
\hline 4ESTADO & $\begin{array}{l}0,155 \\
(0,107)\end{array}$ & $\begin{array}{l}-0,090 \\
(0,350)\end{array}$ & $\begin{array}{l}-0,018 \\
(0,843)\end{array}$ & 1 & & & & & & & \\
\hline 5EXTRAN & $\begin{array}{l}-0,029 \\
(0,796)\end{array}$ & $\begin{array}{c}-0,205^{* *} \\
(0,032)\end{array}$ & $\begin{array}{l}-0,042 \\
(0,658)\end{array}$ & $\begin{array}{l}-0,021 \\
(0,827)\end{array}$ & 1 & & & & & & \\
\hline 6NFINAN & $\begin{array}{l}0,153 \\
(0,111)\end{array}$ & $\begin{array}{c}-0,742^{* * *} \\
(0,000)\end{array}$ & $\begin{array}{l}-0,154 \\
(0,108)\end{array}$ & $\begin{array}{l}-0,076 \\
(0,431)\end{array}$ & $\begin{array}{l}-0,173 * \\
(0,071)\end{array}$ & 1 & & & & & \\
\hline 7FINAN & $\begin{array}{l}0,189 * * \\
(0,048)\end{array}$ & $\begin{array}{c}-0,226^{* *} \\
(0,018)\end{array}$ & $\begin{array}{l}-0,047 \\
(0,626)\end{array}$ & $\begin{array}{l}-0,023 \\
(0,810)\end{array}$ & $\begin{array}{l}-0,052 \\
(0,584)\end{array}$ & $\begin{array}{c}-0,191 * * \\
(0,046)\end{array}$ & 1 & & & & \\
\hline $8 \mathrm{ROE}$ & $\begin{array}{c}0,262 * * * \\
(0,005)\end{array}$ & $\begin{array}{l}-0,003 \\
(0,968)\end{array}$ & $\begin{array}{c}0,067 \\
(0,486)\end{array}$ & $\begin{array}{c}0,032 \\
(0,736)\end{array}$ & $\begin{array}{l}0,163 * \\
(0,090)\end{array}$ & $\begin{array}{l}-0,134 \\
(0,164)\end{array}$ & $\begin{array}{c}0,076 \\
(0,432)\end{array}$ & 1 & & & \\
\hline 9TAMAÑO & $\begin{array}{c}0,652 * * * \\
(0,000)\end{array}$ & $\begin{array}{c}-0,365^{* * *} \\
(0,000)\end{array}$ & $\begin{array}{c}0,074 \\
(0,443)\end{array}$ & $\begin{array}{c}0,077 \\
(0,425)\end{array}$ & $\begin{array}{c}0,021 \\
(0,826)\end{array}$ & $\begin{array}{l}0,229 * * \\
(0,016)\end{array}$ & $\begin{array}{l}0,198^{* *} \\
(0,038)\end{array}$ & $\begin{array}{l}0,178^{*} \\
(0,063)\end{array}$ & 1 & & \\
\hline 10SECTOR & $\begin{array}{l}0,0317 \\
(0,743)\end{array}$ & $\begin{array}{c}-0,211 * * \\
(0,027)\end{array}$ & $\begin{array}{l}0,179 * \\
(0,062)\end{array}$ & $\begin{array}{c}0,139 \\
(0,146)\end{array}$ & $\begin{array}{c}0,131 \\
(0,174)\end{array}$ & $\begin{array}{c}0,020 \\
(0,830)\end{array}$ & $\begin{array}{c}0,092 \\
(0,338)\end{array}$ & $\begin{array}{c}0,044 \\
(0,643)\end{array}$ & $\begin{array}{c}0,004 \\
(0,962)\end{array}$ & 1 & \\
\hline $11 \mathrm{END}$ & $\begin{array}{c}0,153 \\
(0,111)\end{array}$ & $\begin{array}{l}-0,156 \\
(0,104)\end{array}$ & $\begin{array}{l}-0,119 \\
(0,215)\end{array}$ & $\begin{array}{c}0,084 \\
(0,383)\end{array}$ & $\begin{array}{c}0,015 \\
(0,876)\end{array}$ & $\begin{array}{l}0,182 * \\
(0,057)\end{array}$ & $\begin{array}{c}0,002 \\
(0,979)\end{array}$ & $\begin{array}{l}-0,104 \\
(0,280)\end{array}$ & $\begin{array}{c}0,458 * * * \\
(0,000)\end{array}$ & $\begin{array}{c}-0,342 * * * \\
(0,000)\end{array}$ & \\
\hline 12TRANSP & $\begin{array}{l}0,215^{* *} \\
(0,024)\end{array}$ & $\begin{array}{l}-0,053 \\
(0,582)\end{array}$ & $\begin{array}{l}-0,018 \\
(0,850)\end{array}$ & $\begin{array}{c}0,100 \\
(0,298)\end{array}$ & $\begin{array}{c}0,029 \\
(0,762)\end{array}$ & $\begin{array}{c}0,082 \\
(0,391)\end{array}$ & $\begin{array}{l}-0,114 \\
(0,237)\end{array}$ & $\begin{array}{c}0,027 \\
(0,777)\end{array}$ & $\begin{array}{c}0,093 \\
(0,335)\end{array}$ & $\begin{array}{c}0,062 \\
(0,521)\end{array}$ & $\begin{array}{c}0,047 \\
(0,627)\end{array}$ \\
\hline
\end{tabular}

(P-valor) * Estadísticamente significativo al 10\%** Estadísticamente significativo al 5\% $* * *$ Estadísticamente significativo al $1 \%$

Tabla 2. Matriz de correlaciones 
Como paso previo a la estimación del modelo de regresión lineal, se realizó también una comparación entre sub-muestras para ver si existen diferencias estadísticamente significativas en el nivel de transparencia en RSC en función de algunas características organizativas de la empresa. Para dividir la muestra total en dos sub-muestras se calculó el valor mediano de la transparencia en RSC, de manera que, por un lado se agrupan aquellas empresas cuyo índice de transparencia está por encima de la mediana, y por otro lado, aquellas que se sitúan por debajo de la misma. La no normalidad de la variable transparencia en RSC justifica que la mediana sea un indicador de la tendencia central más representativo que la media a la hora de construir los grupos. Dado que el valor mediano de la variable "índice de transparencia en RSC" toma valor cero, las dos sub-muestras se corresponden también con la división de la muestra según el criterio de publicar o no un informe de RSC siguiendo las directrices del GRI.

Así, tal y como muestra la Tabla 3, las empresas con un nivel de transparencia por encima de la mediana en las cuestiones relativas al GRI son más rentables, su tamaño es mayor así como su nivel de endeudamiento y de transparencia informativa. Para confirmar si las diferencias detectadas son estadísticamente significativas, se aplicó la prueba no paramétrica de la $\mathrm{U}$ de Mann-Whitney para dos muestras independientes, dada la no normalidad de las variables consideradas. Acorde con la información proporcionada por el valor mediano, los rangos promedios también indican que las cuatro variables alcanzan valores superiores en la muestra de empresas con un mayor nivel de transparencia informativa en RSC. Además, los resultados obtenidos tras aplicar dicha prueba a partir de los rangos promedios permiten concluir que las diferencias observadas son estadísticamente relevantes y, por ende, no atribuibles al azar, en el caso del nivel de rentabilidad, del tamaño empresarial y de la transparencia informativa. Por otro lado, las empresas con un mayor nivel de transparencia en RSC parecen pertenecer en un mayor porcentaje de los casos a sectores más sensibles a cuestiones medioambientales, aunque de acuerdo con la Chi-cuadrado no parecen existir diferencias significativas entre las dos sub-muestras.

\begin{tabular}{|c|c|c|c|c|c|c|c|}
\hline \multirow[t]{2}{*}{ Variable } & \multicolumn{3}{|c|}{$\begin{array}{l}\text { TRANSPRSC superior mediana } \\
\qquad \mathbf{N}=37\end{array}$} & \multicolumn{3}{|c|}{$\begin{array}{l}\text { TRANSPRSC inferior mediana } \\
\qquad N=72\end{array}$} & \multirow{2}{*}{$\frac{\begin{array}{c}\text { U Mann } \\
\text { Whitney }\end{array}}{U}$} \\
\hline & Media & Mediana & $\mathrm{RP} \mathrm{a}^{\mathrm{a}}$ & Media & Mediana & $\mathrm{RP}$ & \\
\hline ROE & 0,216 & 0,173 & 70,12 & 0,030 & 0,093 & 47,23 & $772,5^{* * *}$ \\
\hline TAMAÑO & $17.463 .613,05$ & 6.017 .000 & 82,85 & $12.77 .970,17$ & $3.93 .420,50$ & 40,69 & $301,5^{* * *}$ \\
\hline END & 0,649 & 0,650 & 60,80 & 0,591 & 0,613 & 52,02 & 1117,5 \\
\hline \multirow[t]{2}{*}{ TRANSP } & 2,49 & 3 & 62,55 & 2,22 & 2 & 51,12 & $1052,5^{* *}$ \\
\hline & \multicolumn{3}{|c|}{$\%$} & \multicolumn{3}{|c|}{$\%$} & Chi cuadrado \\
\hline SECTOR & \multicolumn{3}{|c|}{32,43} & \multicolumn{3}{|c|}{31,94} & 0,003 \\
\hline
\end{tabular}

[a] RP hace referencia al rango promedio de los datos *Significativo estadísticamente al $10 \%$ **Significativo estadísticamente al 5\%***Significativo estadísticamente al $1 \%$

Tabla 3. Diferencias en el nivel de transparencia informativa en RSC en función de las características de la empresa 
A continuación se presentan los resultados relativos a la posible influencia de la identidad del primer gran accionista y de otros determinantes de la transparencia en RSC, resultados obtenidos con el programa STATA9 (Tabla 4). En los modelos planteados se van introduciendo, alternativamente, cada una de las variables relativas a la tipología del primer accionista junto con las variables de control consideradas. Considerando nuestro tamaño muestral, la consideración conjunta de todas las variables puede no ser adecuada, máxime debido a la correlación existente entre varias de las variables proxies de la estructura de propiedad.

En lo que se refiere a las variables explicativas, parece que no existe una influencia estadísticamente significativa de la identidad del primer gran accionista sobre el índice de transparencia en RSC. Solo la presencia de un inversor institucional como primer accionista en el capital de la empresa parece ejercer un efecto negativo y significativo aunque únicamente al nivel del 10 por ciento (Modelo 2, Tabla 4). Estos resultados estarían en línea con los de Coffey y Fryxell, (1991) y Arora y Dharwadkar (2011) para EEUU, quienes afirman que los inversores institucionales están más preocupados por sus intereses a corto plazo que por los beneficios a largo plazo que la inversión en RSC les puedan reportar. Sin embargo, este hallazgo debe ser tomado con cautela dado el reducido nivel de significatividad y teniendo en cuenta que en nuestra muestra el número de observaciones o casos donde el primer accionista es un inversor institucional es reducido. Así pues, podemos concluir que nuestros resultados no parecen apoyar la hipótesis propuesta relativa a la influencia de la naturaleza del primer gran accionista sobre el nivel de transparencia que las empresas tienen sobre sus acciones sociales o en RSC.

De igual forma, en todos los modelos resultan significativas las variables relativas al tamaño, al endeudamiento de la empresa y al nivel general de transparencia de la empresa. En línea con los trabajos de Archel (2003), de Prado-Lorenzo et al. (2009) o de Reverte (2009) para España, de Ghazali (2007) para Malasia o de Castelo y Lima (2008) para Portugal, el tamaño empresarial (TAMAÑO) está asociado de manera positiva con la divulgación de las actividades de naturaleza social que las empresas realizan. Las compañías de más tamaño son las unidades con más capacidad para generar daños sociales y medioambientales y disponen de recursos más adecuados para elaborar la información.

Por su parte, cuanto mayor sea el nivel de endeudamiento (END) menor es el nivel de transparencia en RSC. Una posible explicación es que ante un bajo nivel de endeudamiento de la empresa sus acreedores pueden ejercer menos presión ante sus directivos sobre la realización de actividades de RSC. De manera similar, Castelo y Lima (2008) encuentran que las compañías portuguesas con más endeudamiento son menos transparentes en sus páginas web en lo relativo a sus acciones de RSC. Reverte (2009) para una muestra de empresas españolas del IBEX 35 encontró también un coeficiente negativo de esta variable, aunque no era estadísticamente significativo. 
Además, tal y como a priori era de esperar cuanto mayor sea el nivel de transparencia de una empresa en otros aspectos (por ejemplo, información económica) (TRANSP), mayor será también su divulgación sobre las acciones de naturaleza social que realiza.

\begin{tabular}{|c|c|c|c|c|c|c|}
\hline Variable & 1 & 2 & 3 & 4 & 5 & 6 \\
\hline Constante & $\begin{array}{c}-1,752 * * * \\
(0,258)\end{array}$ & $\begin{array}{c}-1,784 * * * \\
(0,224)\end{array}$ & $\begin{array}{c}-1,734 * * * \\
(0,226)\end{array}$ & $\begin{array}{c}-1,765^{*} \\
(0,227)\end{array}$ & $\begin{array}{c}-1,751 * * * \\
(0,231)\end{array}$ & $\begin{array}{c}-5,203 * * * \\
(0,858)\end{array}$ \\
\hline FAM & $\begin{array}{c}-0,008 \\
(0,063)\end{array}$ & & & & & \\
\hline IINS & & $\begin{array}{c}-0,272 * \\
(0,153)\end{array}$ & & & & \\
\hline ESTADO & & & $\begin{array}{c}0,461 \\
(0,303)\end{array}$ & & & \\
\hline EXTRAN & & & & $\begin{array}{c}-0,120 \\
(0,139)\end{array}$ & & \\
\hline NFINAN & & & & & $\begin{array}{c}0,027 \\
(0,061)\end{array}$ & \\
\hline FINAN & & & & & & $\begin{array}{c}0,124 \\
(0,129)\end{array}$ \\
\hline ROE & $\begin{array}{c}0,141 \\
(0,088) \\
\end{array}$ & $\begin{array}{c}0,144 \\
(0,087) \\
\end{array}$ & $\begin{array}{c}0,135 \\
(0,087) \\
\end{array}$ & $\begin{array}{c}0,153^{*} \\
(0,089) \\
\end{array}$ & $\begin{array}{c}0,147 \\
(0,089) \\
\end{array}$ & $\begin{array}{c}0,138 \\
(0,088)\end{array}$ \\
\hline TAMAÑO & $\begin{array}{c}0,147 * * * \\
(0,018)\end{array}$ & $\begin{array}{c}0,151 * * * \\
(0,017)\end{array}$ & $\begin{array}{c}0,148 * * * \\
(0,017)\end{array}$ & $\begin{array}{c}0,147 * * * \\
(0,017)\end{array}$ & $\begin{array}{c}0,146 * * * \\
(0,018)\end{array}$ & $\begin{array}{c}0,144 * * * \\
(0,018)\end{array}$ \\
\hline SECTOR & $\begin{array}{c}-0,043 \\
(0,068)\end{array}$ & $\begin{array}{c}-0,026 \\
(0,066)\end{array}$ & $\begin{array}{c}-0,059 \\
(0,066)\end{array}$ & $\begin{array}{c}-0,032 \\
(0,067)\end{array}$ & $\begin{array}{c}-0,043 \\
(0,066)\end{array}$ & $\begin{array}{c}-0,046 \\
(0,066)\end{array}$ \\
\hline END & $\begin{array}{c}-0,393 * * \\
(0,193)\end{array}$ & $\begin{array}{c}-0,427 * * \\
(0,190)\end{array}$ & $\begin{array}{c}-0,428^{*} * \\
(0,192)\end{array}$ & $\begin{array}{c}-0,375^{*} \\
(0,192)\end{array}$ & $\begin{array}{c}-0,397 * * \\
(0,192)\end{array}$ & $\begin{array}{c}-0,380 * * \\
(0,192)\end{array}$ \\
\hline TRANSP & $\begin{array}{c}0,097 * * \\
(0,043)\end{array}$ & $\begin{array}{l}0,094 * * \\
(0,043)\end{array}$ & $\begin{array}{l}0,092 * * \\
(0,043)\end{array}$ & $\begin{array}{l}0,098 * * \\
(0,043)\end{array}$ & $\begin{array}{l}0,096 * * \\
(0,043)\end{array}$ & $\begin{array}{l}0,103 * * \\
(0,043)\end{array}$ \\
\hline $\mathbf{F}$ & $16,49 * * *$ & $17,52 * * *$ & $17,24 * * *$ & $16,73 * * *$ & $16,55 * * *$ & $16,79 * * *$ \\
\hline $\mathbf{N}$ & 109 & 109 & 109 & 109 & 109 & 109 \\
\hline $\mathbf{R}^{2}$ & 0,492 & 0,507 & 0,503 & 0,496 & 0,493 & 0,496 \\
\hline
\end{tabular}

[a] Error estándar entre paréntesis

*Estadísticamente significativo al $10 \%$

$* *$ Estadísticamente significativo al $5 \%$

$* * *$ Estadísticamente significativo al $1 \%$

Tabla 4. Determinantes de la transparencia en RSC [a]

En lo que se refiere al nivel de rentabilidad empresarial ésta solo parece afectar a la transparencia informativa en RSC en uno de los modelos y únicamente al nivel del 10\%. Este resultado es similar al mostrado por estudios previos para el caso español, como por ejemplo, Archel (2003), Carmona y Carrasco (1988), Moneva y Llena (1996) o Reverte (2009) -que no encuentran una relación significativa-. Finalmente, el sector de actividad de la empresa (SECTOR), a diferencia de otros estudios previos (incluido el de Reverte, 2009 o Prado-Lorenzo et al., 2009 para España), pero al igual que Ghazali (2007) para una muestra de empresas cotizadas en Malasia, no parece influir de manera estadísticamente significativa. El mayor porcentaje de empresas de "sectores más sensible a cuestiones medioambientales" en el trabajo de Reverte (2009) para las 35 empresas del IBEX 35 o la consideración de varias variables dummies según el sector de actividad en el caso de Prado-Lorenzo et al. (2009) 
puede explicar la diferencia entre nuestros resultados y los estudios previos para el caso español.

Aunque no se muestran los resultados, es necesario mencionar que hemos repetido las estimaciones considerando la rentabilidad económica (beneficio de explotación / activo total) y un indicador de la performance no contable como el valor empresarial (definido como el cociente entre el valor de mercado o capitalización de la empresa más el valor contable de la deuda dividido por el valor contable de los fondos propios) en lugar de la rentabilidad financiera, y los resultados no varían en lo que se refiere a las principales variables explicativas. Además, hemos considerado la tipología del primer accionista teniendo en cuenta si éste tiene una participación significativa en el capital de la empresa de acuerdo con lo establecido por la CNMV (porcentaje en el capital superior al 3 por ciento). Las estimaciones se ven reducidas solamente en una observación y no varían tanto en lo que se refiere a las variables explicativas como a las variables de control.

\section{Conclusiones}

En los últimos años, la realización de actividades de RSC se ha convertido en una práctica que, con relativa frecuencia, las empresas están utilizando de modo voluntario con la finalidad de mejorar las condiciones sociales y medioambientales en sus negocios, así como su relación con los distintos stakeholders, pues la RSC va más allá del cumplimiento de las leyes y las normas, dando por supuesto su respeto y estricto cumplimiento. Bajo este concepto se engloban un conjunto de prácticas, estrategias y sistemas de gestión empresariales que persiguen un nuevo equilibrio entre las dimensiones económica, social y ambiental.

Además, los estudios longitudinales sobre información social corporativa ponen de manifiesto un incremento paulatino del número de empresas que publican información sobre las acciones de RSC que desarrollan y de su contenido informativo (Archel, 2003; Deegan \& Gordon, 1996; Moneva \& Llena, 2000). La transparencia en RSC ayuda a reducir asimetrías informativas entre la compañía y sus stakeholders al proporcionar información relevante a agentes externos a la compañía a la vez que influye en sus percepciones y los proyectos financieros del futuro. Varios estudios han mostrado que este comportamiento informativo está influenciado por una variedad de características propias de las empresas y de la industria en la que están presentes (Cormier \& Magnan, 2003).

En este sentido, después de controlar por ciertos rasgos organizativos tales como el nivel de rentabilidad, el tamaño, el sector de actividad o el endeudamiento y tras aplicar un análisis de corte transversal mediante un modelo de regresión lineal a una muestra de compañías españolas cotizadas en el año 2008, los hallazgos han puesto de manifiesto que solo la presencia de un inversor institucional como gran accionista en el capital de la empresa parece ejercer un efecto negativo y significativo en la divulgación de información sobre las acciones de RSC. Una posible explicación es que este tipo de inversores pueden estar más preocupados por 
sus intereses a corto plazo que por los beneficios a largo plazo que las inversiones y transparencia en RSC les pueden reportar. En todo caso, dado el bajo nivel de significatividad y el reducido número de casos de nuestra muestra donde un inversor institucional es el primer accionista, este resultado debe ser tomado con cautela. Así pues, en conjunto podemos decir que no hemos encontrado apoyo para la hipótesis planteada relativa a una influencia significativa de la identidad del primer gran accionista sobre la transparencia en RSC.

Por otra parte, las empresas de mayor tamaño empresarial, aquellas con un menor nivel de endeudamiento y que en general presentan un mayor nivel de transparencia parecen realizar una mayor divulgación de información sobre las acciones sociales realizadas. Así pues, nuestros resultados corroboran los de estudios previos, incluidos algunos para el caso español, que apuntaban que determinadas características propias de la empresa influyen en su nivel de transparencia sobre información social.

Como limitación a la hora de interpretar los resultados obtenidos cabe mencionar que somos conscientes de que pueden existir otros indicadores de transparencia, como por ejemplo, la información de contenido social y medioambiental en los informes anuales de las empresas. Además, hemos trabajado con una muestra de un único país y en un momento temporal concreto. Por ello, en futuras investigaciones se podrían añadir empresas de otros países y/o aplicar un análisis de datos de panel para comprobar la existencia o no de relaciones en el medio o largo plazo así como para corregir de manera más precisa, gracias a la utilización de técnicas econométricas más robustas, el problema de endogeneidad entre la transparencia en RSC y determinadas características empresariales. De igual forma, puede ser interesante analizar no solo la influencia no del primer gran accionista de la empresa sino del último gran propietario, teniendo en cuenta para el caso español la existencia de cadenas de propiedad.

\section{Agradecimientos}

Los autores agradecen la ayuda financiera recibida de la Consejería de Educación de la Junta de Castilla y León (proyecto LE004A10-1) y del Ministerio de Economía y Competitividad (proyectos ECO2012-36532, ECO2012-35439), así como los comentarios y sugerencias recibidos por los evaluadores anónimos que han contribuido a mejorar la versión inicial del trabajo.

\section{Referencias}

ADAMS, C.A.; HILL, W.Y.; ROBERTS, C.B. (1998). Corporate social reporting practices in Western Europe: Legitimating corporate behaviour. The British Accounting Review, 30(1): 121. http://dx.doi.org/10.1006/bare.1997.0060

AGLE， B.R.; MITCHELL, R.K.; SONNENFELD, J.A. (1999). Who matters to CEOs? An investigation of stakeholder attributes and salience, corporate performance, and CEO values. Academy of Management Journal, 42(5): 507-525. ttp://dx.doi.org/10.2307/256973 
AGUILERA, R.; RUPP, D.; WILLIAMS, C.; GANAPATHI, J. (2007). Putting the back in Corporate Social Responsibility: a multilevel theory of social change in organizations. The Academy of Management Review, 32(3): 836-863. http://dx.doi.org/10.5465/AMR.2007.25275678

AGUILERA, R.; WILLIAMS, C.; CONLEY, J.M.; RUPP, D.E. (2006). Corporate governance and social responsibility: A comparative analysis of the UK and the US. Corporate Governance: An International Review, 14: 147-158.

AKERLOF, G.A. (1970). The market for 'Lemons': Quality uncertainty and the market mechanism. Quarterly Journal of Economics, 84: 488-500. http://dx.doi.org/10.2307/1879431

ALBINGER, H.S.; FREEMAN, S. J. (2000). Corporate social performance and attractiveness as an employer to different job seeking populations. Journal of Business Ethics, 28(3): 243253. http://dx.doi.org/10.1023/A:1006289817941

ANDERSON, R.C.; MANSI, S.A.; REEB, D.M. (2003). Founding family ownership and the agency cost of debt. Journal of Financial Economics, 68: 263-285. http://dx.doi.org/10.1016/S0304405X(03)00067-9

ANDERSON, R.C.; REEB, D.M. (2003). Founding-family ownership and firm performance: Evidence from the S\&P 500. The Journal of Finance, 58(3): 1301-1328. http://dx.doi.org/10.1111/1540-6261.00567

ANDREW, B.H.; GUL, F.A.; GUTHRIE, J.E.; TEOH, H.Y. (1989). A note on corporate social disclosure practices in developing countries: The case of Malaysia and Singapore. British Accounting Review, 21(4): 371-376. http://dx.doi.org/10.1016/0890-8389(89)90034-6

ARCHEL, P. (2003). La divulgación de la información social y medioambiental de la gran empresa española en el período 1994-1998: Situación actual y perspectivas. Revista Española de Financiación y Contabilidad, 117: 571-601.

ARORA, P.; DHARWADKAR, R. (2011). Corporate governance and corporate social responsibility (CSR): The moderating roles of attainment discrepancy and organization snack. Corporate Governance: An International Review, 19(2): 136-152. http://dx.doi.org/10.1111/j.14678683.2010.00843.x

BAGNOLI, M.; WATTS, S.G. (2003). Selling to socially responsible consumers: Competition and the private provision of public goods. Journal of Economics \& Management Strategy, 12(3): 419-445. http://dx.doi.org/10.1162/105864003322309536

BALABANIS, G.; PHILLIPS, H.C.; LYALL, J. (1998). Corporate social responsibility and economic performance in the top British companies: Are they linked? European Business Review, 98(1): 25-44. http://dx.doi.org/10.1108/09555349810195529 
BARNEA, A.; RUBIN, A. (2010). Corporate social responsibility as a conflict between shareholders. Journal of Business Ethics, 97(1): 71-86. http://dx.doi.org/10.1007/s10551-0100496-z

BECHT, M.; RÖELL, A. (1999). Blockholdings in Europe: An international comparison. European Economic Review, 43: 1049-1056. http://dx.doi.org/10.1016/S0014-2921(98)00113-5

BELKAOUI, A.; KARPIK, P.G. (1989). Determinants of the corporate decision to disclose social information. Accounting, Auditing and Accountability Journal, 2(1): 36-51. http://dx.doi.org/10.1108/09513578910132240

BERRONE，P.; CRUZ，C.; GÓMEZ-MEJÍA， L.R.; LARRAZA-KINTANA, M. (2010). Socioemotional wealth and corporate responses to institutional pressures: Do family-controlled firms pollute less? Administrative Science $\quad$ Quarterly, 52-113. http://dx.doi.org/10.2189/asqu.2010.55.1.82

BLACKBURN, RM.; JARMAN, J.; SILTANEN, J. (1994). A reply to Lampard. Work Employment \& Society, 8: 413-419.

BLAIR, M. (1995). Ownership and control. Rethinking governance for the twenty-first century. Washington DC: The Brooking Institution.

BRAMMER, S.; PAVELIN, S. (2006). Voluntary environmental disclosure by large UK companies. Journal of Business Finance and Accounting, 33(7/8): 1168-1188. http://dx.doi.org/10.1111/j.1468-5957.2006.00598.x

BRAMMER, S.; PAVELIN, S. (2008). Factors influencing the quality of corporate environmental disclosure. Business Strategy and the Environment, 17: 120-136. http://dx.doi.org/10.1002/bse.506

BROWN, B.; PERRY, S. (1994). Removing the Financial Performance Halo from Fortune's 'Most Admired' Companies. Academy of Management Journal, 37: 1347-1359. http://dx.doi.org/10.2307/256676

BROWN, N.; DEEGAN, C. (1998). The public disclosure of environmental performance information. A dual test of media agenda setting theory and legitimacy theory. Accounting and Business Research, 29(1): 21-41. http://dx.doi.org/10.1080/00014788.1998.9729564

BURAK, A.; MORANTE, L.S. (2007). Corporate social responsibility and firm characteristics in Sweden: Who and what makes a firm a better corporate citizen? Master's Thesis in Finance Stockholm School of Economics.

BURKE, L.; LOGSDON, JM.; MITCHELL, W.; REINER, M.; VOGEL, D. (1986). Corporate community involvement in the San Francisco Bay Area. California Management Review, 28(3): 122-141. http://dx.doi.org/10.2307/41165206 
BUSHEE, B. (1998). The influence of institutional investors on myopic R\&D investment behaviour. The Accounting Review, 73: 305-333.

BUSHEE, B. (2001). Do institutional investors prefer near-term earnings over long-run value? Contemporary Accounting Research, 18(2): 207-246. http://dx.doi.org/10.1506/J4GU-BHWH8HME-LEOX

CAMPBELL, D. (2004). A longitudinal and cross-sectional analysis of environmental disclosure in UK companies -a research note. British Accounting Review, 36: 107-117. http://dx.doi.org/10.1016/j.bar.2003.09.001

CARMONA, S.; CARRASCO, F. (1988). Información de contenido social y estados contables: Una aproximación empírica y algunas consideraciones teóricas. Actualidad Financiera, 11: 2175-2192.

CASTELO, M.; LIMA, L. (2008). Factors influencing social responsibility disclosure by Portuguese companies. Journal of Business Ethics, 83: 685-701. http://dx.doi.org/10.1007/s10551-007-9658-z

CHIH, H.; CHIH, H.; CHEN, T. (2010). On the determinants of corporate social responsibility: International evidence on the financial industry. Journal of Business Ethics, 93: 115-135. http://dx.doi.org/10.1007/s10551-009-0186-x

CLARKE， J.; GIBSON-SWEET, M. (1999). The use of corporate social disclosures in the management of reputation and legitimacy: A Cross sectorial analysis of UK top 100 companies. Business Ethics: An European Review, 8(1): 5-13. http://dx.doi.org/10.1111/14678608.00120

COFFEY, B.; FRYXELL, G.E. (1991). Institutional ownership of stock and dimensions of corporate social performance: An empirical examination. Journal of Business Ethics, 10: 437-444. http://dx.doi.org/10.1007/BF00382826

CONSOLANDI, C.; NASCENZI, P.; JAISWAL-DALE, A. (2008). Ownership concentration and corporate social performance: An empirical evidence for European firms. Corporate Responsibility Research Conference 2008, Belfast.

CORMIER, D.; MAGNAN, M. (2003). Environmental reporting management: a European perspective. Journal of Accounting and Public Policy, 22(1): 43-62. http://dx.doi.org/10.1016/S0278-4254(02)00085-6

CORMIER, D.; MAGNAN, M.; VAN VELTHOVEN, B. (2005). Environmental disclosure quality in large German companies: Economic incentives, public pressures or institutional conditions? European Accounting Review, 14(1): 3-39. http://dx.doi.org/10.1080/0963818042000339617 
COWEN, S.S.; FERRERI, L.B.; PARKER, L.D. (1987). The impact of corporate characteristics on social responsibility disclosure: A typology and frequency-based analysis. Accounting, Organizations \& Society, 12(2): 111-122. http://dx.doi.org/10.1016/0361-3682(87)90001-8

CULLEN, L.; CHRISTOPHER, T. (2002). Governance disclosures and firm characteristics of listed Australian mining companies. International Journal of Business Studies, 10(1): 37-58.

DEEGAN, C. (2002). The legitimising effect of social and environmental disclosures-A theoretical foundation. Accounting, Auditing and Accountability Journal, 15(3): 282-311. http://dx.doi.org/10.1108/09513570210435852

DEEGAN, C.; GORDON, B. (1996). A study of the environmental disclosure practices of Australian corporations. Accounting and Business Research, 26(3): 187-199. http://dx.doi.org/10.1080/00014788.1996.9729510

DEEGAN, C.; RANKIN, M. (1996). Do Australian companies objectively report environmental news? An analysis of environmental disclosures by firms succeefully prosecuted by the environmental protection authority. Accounting, Auditing and Accountability Journal, 9(2): 50-67. http://dx.doi.org/10.1108/09513579610116358

DÉNIZ, M.C.; CABRERA, M.K. (2005). Corporate social responsibility and family business in Spain. Journal of Business Ethics, 56(1): 27-41. http://dx.doi.org/10.1007/s10551-004-3237-3

DILLER, J. (1999). A social conscience in the global marketplace? Labour dimensions of codes of conduct, social labelling and investor initiatives. International Labour Review, 138(2): 99129. http://dx.doi.org/10.1111/j.1564-913X.1999.tb00062.x

DOWLING, J.; PFEFFER, J. (1975). Organisational legitimacy: Social values and organisational behaviour. Pacific Sociological Review, 18(1): 122-136. http://dx.doi.org/10.2307/1388226

ENG, L.L.; MAK, Y.T. (2003). Corporate governance and voluntary disclosure. Journal of Accounting and Public Policy, 22: 325-345. http://dx.doi.org/10.1016/S0278-4254(03)00037-1

FACCIO, M.; LANG, L.; YOUNG, L. (2001). Dividends and expropriation. American Economic Review, 91(1): 54-71. http://dx.doi.org/10.1257/aer.91.1.54

FREEMAN, E.R.; PARMAR, B.D.; HARRISON, J.S.; WICKS, A.C.; PURNELL, L.; COLLE, S. (2010). Stakeholder theory: The state of the art. The Academy of Management Annals, 4(1): 403-445. http://dx.doi.org/10.1017/СВ09780511815768

FREEMAN, R.E. (1984). Strategic Management: A Stakeholder Approach. Boston: Pitman Publishing Inc.

FRIEDMAN, M. (1962). Capitalism and Freedom. Chicago: Chicago University Press. 
GAMBLE, G.; HSU, K.; JACKSON, C.; TOLLERSON, C. (1996). Environmental disclosures in annual reports: An international perspective. International Journal of Accounting, 31(3): 293-331. http://dx.doi.org/10.1016/S0020-7063(96)90022-9

GARCÍA-AYUSO, M.; LARRINAGA, C. (2003). Environmental disclosure in Spain: Corporate characteristics and media exposure. Spanish Journal of Finance and Accounting, 115: 184214.

GHAZALI, N. (2007). Ownership structure and corporate social responsibility disclosure: Some Malaysian evidence. Corporate Governance, 7(3): 251-266. http://dx.doi.org/10.1108/14720700710756535

GINER, B. (1997). The Influence of company characteristics and accounting regulation on information disclosed by Spanish firms. European Accounting Review, 6(1): 45-68. http://dx.doi.org/10.1080/096381897336863

GODOS-DÍEZ, J.L.; FERNÁNDEZ-GAGO, R.; CABEZA-GARCÍA， L. (2012). Influencia de la propiedad y el control en la puesta en práctica de la RSC en las grandes empresas españolas. Cuadernos de Dirección y Economía de la Empresa, 15(1): 1-11. http://dx.doi.org/10.1016/j.cede.2011.06.002

GÓMEZ-MEJÍA, L.R.; TAKÁCS HAYNES, K.; NÚÑEZ-NICKEL, M.; JACOBSON, KJL.; MOYANOFUENTES, J. (2007). Socioemotional wealth and business risks in family-controlled firms: Evidence from Spanish olive oil mills. Administrative Science Quarterly, 52: 106-137.

GRAAFLAND, J.J. (2002). Corporate social responsibility and family business. Paper presented at the Research Forum of the Family Business Network 13th Annual Conference. Helsinki, Finland.

GRAVES, S.B.; WADDOCK, S.A. (1994). Institutional owners and corporate social performance. Academy of Management Journal, 37(4): 1034-1046. http://dx.doi.org/10.2307/256611

GRAY, R.; JAVAD, M.; POWER, D.M.; SINCLAIR, CD. (2001). Social and environmental disclosure and corporate characteristics: a research note and extension. Journal of Business Finance and Accounting, 28(3/4): 327-356. http://dx.doi.org/10.1111/1468-5957.00376

GRAY, R.; KOUHY, R.; LAVERS, S. (1995). Corporate social and environmental reporting: a review of the literature and a longitudinal study of UK disclosure. Accounting, Auditing and Accountability Journal, 8(2): 47-77. http://dx.doi.org/10.1108/09513579510146996

GRAY, R.; OWEN, D.; ADAMS, C. (1996). Accounting and Accountability. Changes and Challenges in Corporate Social and Environmental Reporting. Londres: Prentice-Hall.

GUTHRIE, J.; PARKER, L.D. (1989). Corporate social reporting: a rebuttal of legitimacy theory. Accounting and Business Research, 343-352. http://dx.doi.org/10.1080/00014788.1989.9728863 
GUTHRIE, J.; PARKER, L.D. (1990). Corporate social disclosure practice: a comparative international analysis. Advances in Public Interest Accountancy, 3: 159-176.

HACKSTON, D.; MILNE, M. (1996). Some determinants of social and environmental disclosures in New Zealand companies. Accounting, Auditing and Accountability Journal, 9(1): 77-108. http://dx.doi.org/10.1108/09513579610109987

HAMID, A. (2004). Corporate social disclosure by banks and finance companies. Corporate Ownership and Control, 1(4): 118-130.

HANIFFA, R.M.; COOKE, T.E. (2005). The impact of culture and governance on corporate social reporting. Journal of Accounting and Public Policy, 24: 391-430. http://dx.doi.org/10.1016/j.jaccpubpol.2005.06.001

HARJOTO, M.A.; JO, H. (2008). Corporate social responsibility and operating performance. Journal of Academy of Business and Economics, 8(1): 59-71.

HECKMAN, J. (1979). Sample selection bias as a specification error. Econométrica, 47:153161. http://dx.doi.org/10.2307/1912352

HILLMAN, A.J.; KEIM, G.D. (2001). Source shareholder value, stakeholder management, and social issues: What's the bottom line? Strategic Management Journal, 22(2): 125-139. http://dx.doi.org/10.1002/1097-0266(200101)22:2<125: :AID-SMJ150>3.0.CO;2-H

HOOGHIEMSTRA, R. (2000). Corporate communication and impression management - new perspectives why companies engage in corporate social reporting. Journal of Business Ethics, 27: 55-68. http://dx.doi.org/10.1023/A:1006400707757

HOOPES, D.G.; MILLER, D. (2006). Ownership preferences, competitive heterogeneity, and family-controlled businesses. Family Business Review, 19(2): 89-101. http://dx.doi.org/10.1111/j.1741-6248.2006.00064.x

HOSSAIN, M.; PERERA, M.B.; RAHMAN, AR. (1995). Voluntary disclosure in the annul reports of New Zealand companies. Journal of International Financial Management and Accounting, 6(1): 69-87. http://dx.doi.org/10.1111/j.1467-646X.1995.tb00050.x

HUAFANG, X.; JIANGUO, Y. (2007). Ownership structure, board composition and corporate voluntary disclosure. Managerial Auditing Journal, 22(6): 604-619. http://dx.doi.org/10.1108/02686900710759406

ISMAIL, K.N.; CHANDLER, R. (2005). Disclosure in the quarterly reports of Malaysian companies. Financial Reporting, Regulation and Governance, 4(1): 1-26.

JENKINS, H.M.; YAKOVLEVA, N. (2006). Corporate social responsibility in the mining industry: Exploring trends in social and environmental disclosure. Journal of Cleaner Production, 14(3/4): 271-284. http://dx.doi.org/10.1016/j.jclepro.2004.10.004 
JENSEN, M.; MECKLING, W. (1976). Theory of the firm: managerial behaviour, agency costs and ownership structure. Journal of Financial Economics, 3(4): 305-360. http://dx.doi.org/10.1016/0304-405X(76)90026-X

JOHNSON, R.A.; GREENING, D.W. (1999). The effects of corporate governance and institutional ownership types on corporate social performance. Academy of Management Journal, 42(5): 564-576. http://dx.doi.org/10.2307/256977

KNOX, S.; MAKLAN, S.; FRENCH, P. (2006). Corporate social responsibility: Exploring stakeholder relationships and programme reporting across leading FTSE companies. Journal of Business Ethics, 61(1): 7-28. http://dx.doi.org/10.1007/s10551-005-0303-4

KUO, L.; YEH, CH.; YU, H. (2012). Disclosure of corporate social responsibility and environmental management: Evidence from China. Corporate Social Responsibility and Environmental Management, 19: 273-287. http://dx.doi.org/10.1002/csr.274

LA PORTA, R.; LOPEZ-DE-SILANES, F.; SHLEIFER, A. (1999). Corporate ownership around the world. The Journal of Finance, 54: 471-517. http://dx.doi.org/10.1111/0022-1082.00115

LENOX, M.; NASH, J. (2003). Industry self-regulation and adverse selection: A comparison across four trade association programs. Business Strategy and Environment, 12(6): 343356. http://dx.doi.org/10.1002/bse.380

LEV, B.; PETROVITIS, C.; RADHAKRISHNAN. S. (2006). Is Doing Good Good for You: Yes, Charitable Contributions Enhances Revenue Growth. New York: University Stern School of Business.

LI, W.; ZHANG, R. (2010). Corporate social responsibility, ownership structure, and political interference: Evidence from China. Journal of Business Ethics, 96(4): 631-645. http://dx.doi.org/10.1007/s10551-010-0488-z

LINE, M.; HAWLEY, H.; KRUT, R. (2002). Development in global environmental and social reporting. Corporate Environmental Strategy, 9(1): 69-78. http://dx.doi.org/10.1016/S10667938(01)00159-2

LÓPEZ-ITURRIAGA, F.; LOPEZ-DE-FORONDA, O. (2011). Corporate social responsibility and reference shareholders: An analysis of European firms. Transnational Corporations Review, 3(3): 1-11.

MAHAPATRA, S. (1984). Investor reaction to corporate social accounting. Journal of Business Finance and Accounting, 11(1): 29-40. http://dx.doi.org/10.1111/j.1468-5957.1984.tb00054.x

MAZURKIEWIC, P. (2006). Corporate Environmental Responsibility: Is a Common CSR Framework Possible? Working Paper del Banco Mundial.

MCGUIRE, JB.; SUNDGREN, A.; SCHNEEWEIS, T. (1988). Corporate social responsibility and firm financial performance. The Academy of Management Journal, 31: 854-872. 
MCVEY, H.; DRAHO, J. (2005). U.S. family-run companies - They may be better than you think. Journal of Applied Corporate Finance, 17(4): 134-143. http://dx.doi.org/10.1111/j.17456622.2005.00067.x

MCWILLIAMS, A.; SIEGEL, DS. (2000). Corporate Social Responsibility and Financial Performance: Correlation or Misspecification? Strategic Management Journal, 21: 603-609. http://dx.doi.org/10.1002/(SICI)1097-0266(200005)21:5<603: :AID-SMJ101>3.0.CO;2-3

MILLER, D.; LE BRETON-MILLER, I. (2006). Family governance and firm performance: Agency, stewardship, and capabilities. Family Business Review, 19: 73-87. http://dx.doi.org/10.1111/j.1741-6248.2006.00063.x

MITCHELL, R.K.; AGLE, B.R.; WOOD, D.J. (1997). Toward a theory of stakeholder identification and salience: Defining the principle of whom and what really counts. Academy of Management Review, 22: 853-886.

MONEVA, J.M.; LLENA, F. (1996). Análisis de la información sobre responsabilidad social en las empresas industriales que cotizan en bolsa. Revista Española de Financiación y Contabilidad, 25(87): 361-402.

MONEVA, J.M.; LLENA, F. (2000). Environmental disclosures in the annual reports of large companies in Spain. European Accounting Review, 9(1): 7-29. http://dx.doi.org/10.1080/096381800407923

MONKS, R.; MINOW, N. (1995). Corporate Governance. England: Cambridge MA.

MORCK, R.; WOLFENZON, D.; YEUNG, B. (2005). Corporate governance, economic entrenchment, and growth. Journal of Economic Literature, 43: 655-720. http://dx.doi.org/10.1257/002205105774431252

NDEMANGA, DA.; KOFFI, E.T. (2009). Ownership structure, industry sector and corporate social responsibility (CSR) practices: - The case of Swedish listed companies. Master of Science in Accounting, Master Degree Project No. 2009: 31.

NESS, K.; MIRZA, A. (1991). Corporate social disclosure: A note on the test of agency theory. The British Accounting Review, 23: 211-217. http://dx.doi.org/10.1016/0890-8389(91)90081-C

NEU, D.; WARSAMHE, H.; PEDWELL, K. (1998). Managing public impressions: environmental disclosures in annual reports. Accounting, Organizations and Society, 23(3): 265-282. http://dx.doi.org/10.1016/S0361-3682(97)00008-1

NEUBAUM, D.O.; ZAHRA, S.A. (2006). Institutional ownership and corporate social performance: The moderating effects of investment horizon, activism, and coordination. Journal of Management, 32: 108. http://dx.doi.org/10.1177/0149206305277797

ORLITZKY, M.; SCHMIDT, F.; RYNES, S. (2003). Corporate social and financial performance. Organization Studies, 24(3): 403-411. http://dx.doi.org/10.1177/0170840603024003910 
ORTIZ DE MANDOJANA, N.; ARAGÓN, A.; DELGADO, J. (2011). La relación entre la propiedad industrial y de los directivos y el desempeño medioambiental. Cuadernos de Economía y Dirección de Empresa, 10: 1016. http://dx.doi.org/10.1016/j.cede.2010.10.001

PATTEN, D. (1991). Exposure, legitimacy, and social disclosure. Journal of Accounting and Public Policy, 10: 297-308. http://dx.doi.org/10.1016/0278-4254(91)90003-3

PATTEN, D. (1992). Intra-Industry environmental disclosures in response to the Alaskan oil spill: A note on legitimacy theory. Accounting, Organizations and Society, 17(5): 471-475. http://dx.doi.org/10.1016/0361-3682(92)90042-Q

PATTEN, D.M. (2002a). Give or take on the Internet: An examination of the disclosure practices of insurance firm web innovators. Journal of Business Ethics, 36(3): 247-259. http://dx.doi.org/10.1023/A:1014009229437

PATTEN, D.M. (2002b). Media exposure, public policy pressure, and environmental disclosure: An examination of the impact of tri data availability. Accounting Forum, 26(2): 152-171. http://dx.doi.org/10.1111/1467-6303.t01-1-00007

PAVA, L.; KRAUSZ, J. (1996). The association between corporate social-responsibility and financial performance: The Paradox of social Cost. Journal of Business Ethics, 15: 321-357. http://dx.doi.org/10.1007/BF00382958

PERROW, C. (1970). Organizational Analysis: A sociological view. Wadsworth: Belmont CA.

PORTER, M.E. (1992). Capital choices: Changing the way America invests in industry. Journal of Applied Corporate Finance, 5(2): 4-16. http://dx.doi.org/10.1111/j.1745-6622.1992.tb00485.x PORTER, M.E.; KRAMER, M.R. (2002). The competitive advantage of corporate philanthropy. Harvard Business Review, 80(12): 56-68.

PRADO-LORENZO， J.M.; GARCÍA-SÁNCHEZ， I.M.; GALLEGO-ÁLVAREZ， I. (2009). Características del consejo de administración e información en materia de Responsabilidad Social Corporativa. Revista Española de Financiación y Contabilidad, 141: 107-135.

PRENCIPE, A. (2004). Proprietary costs and determinants of voluntary segment disclosure: Evidence from Italian Listed Companies. European Accounting Review, 13(2): 319-340. http://dx.doi.org/10.1080/0963818042000204742

PRESTON, L.; SAPIENZA, H.; MILLAR, R. (1991). Stakeholder, shareholders, managers: Who gains from corporate performance? en Etzioni, A. y Lawrence, P. (eds.). Socio-Economics: Towards a new synthesis, Armonk: M.E. Sharpe.

PRESTON, L.E.; O'BANNON, D. (1997). The corporate social-financial performance relationship. Business \& Society, 36: 5-31. http://dx.doi.org/10.1177/000765039703600406 
REVERTE, C. (2009). Determinants of corporate social responsibility disclosure ratings by Spanish listed firms. Journal of Business Ethics, 88: 351-366. http://dx.doi.org/10.1007/s10551-008-9968-9

ROBERTS, R.W. (1992). Determinants of corporate social responsibility disclosure: An application of stakeholder theory. Accounting, Organizations \& Society, 17(6): 595-612. http://dx.doi.org/10.1016/0361-3682(92)90015-K

RUHNKA, J.; BOERSTLER, H. (1998). Governmental incentives for corporate self-regulation. Journal of Business Ethics, 17(3): 309-326. http://dx.doi.org/10.1023/A:1005757628513

SCHULZE, W.S.; LUBATKIN, M.H.; DINO, R.N. (2003). Toward a theory of agency and altruism in family firms. Journal of Business Venturing, 18: 473-450. http://dx.doi.org/10.1016/S08839026(03)00054-5

SEE, G. (2009). Harmonious society and Chinese CSR: Is there really a link? Journal of Business Ethics, 89(1): 1-22. http://dx.doi.org/10.1007/s10551-008-9981-z

SHARMA, P.; CHRISMAN, J.J.; CHUA, J.H. (1997). Strategic management of the family business: Past research and future challenges. Family Business Review, 10: 1-35. http://dx.doi.org/10.1111/j.1741-6248.1997.00001.x

SINGH, D.R.; AHUJA, J.M. (1983). Corporate social reporting in India. The International Journal of Accounting, Spring: 151-69.

SPICER, B.H. (1978). Investors, corporate social performance, and information disclosure: An empirical study. The Accounting Review, 53: 94-111.

STANWICK, P.; STANWICK, S. (1998). The relationship between corporate social performance and organization size, financial performance and environmental performance: An empirical examination. Journal of Business Ethics, 17: 195-204. http://dx.doi.org/10.1023/A:1005784421547

TURBAN, D.B.; GREENING, D.W. (1997). Corporate social performance and organizational attractiveness to prospective employees. Academy of Management Journal, 40: 658-672. http://dx.doi.org/10.2307/257057

ULLMANN, A. (1985). Data in search of a theory: A critical examination of the relationships among social performance, social disclosure, and economic performance of U.S. firms. Academy of Management Review, 10(3): 540-557.

VILLIERS, C.; NAIKER, V.; STADEN, C.J. (2011). The effect of board characteristics on firm environmental performance. Journal of Management, 37(6): 1636-1663. http://dx.doi.org/10.1177/0149206311411506 
WADDOCK, S.; GRAVES, S.B. (1997). The corporate social performance- financial performance link. Strategic Management Journal, 18(4): 303-319. http://dx.doi.org/10.1002/(SICI)10970266(199704)18:4<303: :AID-SMJ869>3.0.CO;2-G

WALLACE, R.; NASER, K.; MORA, A. (1994). The relationship between the comprehensiveness of corporate annual reports and firm characteristics in Spain. Accounting and Business Research, 25(97): 41-53. http://dx.doi.org/10.1080/00014788.1994.9729927

WATTS, R.L.; ZIMMERMAN, J.L. (1986). Positive Accounting Theory. Englewood Cliffs: PrenticeHall.

WOTRUBA, T. (1997). Industry self-regulation: A review and extension to a global setting. Journal of Public Policy \& Marketing, 16(1): 38-54.

WU, M.L. (2006). Corporate social performance, corporate financial performance and firm size: A meta-analysis. Journal of American Academy of Business, 8: 163-171.

ZENG, S.X.; XU, X.D.; YIN, H.T.; TAM, C.M. (2012). Factors that drive Chinese listed companies in voluntary disclosure of environmental information. Journal of Business Ethics, 109: 309-321. http://dx.doi.org/10.1007/s10551-011-1129-x

\section{Apéndice 1}

\begin{tabular}{|c|c|}
\hline $\begin{array}{c}\text { Variables de la } \\
\text { dimensión perfil }\end{array}$ & Número de indicadores \\
\hline Estrategia y Análisis & 2 \\
\hline Perfil de la Organización & 10 \\
\hline Parámetros del Informe & 13 \\
\hline $\begin{array}{c}\text { Gobierno, Participación y } \\
\text { compromisos con los } \\
\text { Grupos de Presión }\end{array}$ & 17 \\
\hline $\begin{array}{c}\text { Variables de la } \\
\text { dimensión económica }\end{array}$ & Número de indicadores \\
\hline Desempeño Económico & 4 \\
\hline Presencia en el Mercado & 3 \\
\hline $\begin{array}{c}\text { Impactos Económicos } \\
\text { Indirectos }\end{array}$ & 2 \\
\hline $\begin{array}{c}\text { Variables de la } \\
\text { dimensión ambiental }\end{array}$ & Número de indicadores \\
\hline Materiales & 2 \\
\hline Energía & 5 \\
\hline Agua & 3 \\
\hline Biodiversidad & 5 \\
\hline
\end{tabular}




\begin{tabular}{|c|c|}
\hline $\begin{array}{c}\text { Emisiones, vertidos y } \\
\text { residuos }\end{array}$ & 10 \\
\hline Productos y Servicios & 2 \\
\hline Cumplimiento normativo & 1 \\
\hline Transporte & 1 \\
\hline General & 1 \\
\hline $\begin{array}{c}\text { Variables de la } \\
\text { dimensión social }\end{array}$ & Número de indicadores \\
\hline $\begin{array}{c}\text { Prácticas Laborales y } \\
\text { Éticas }\end{array}$ & 11 \\
\hline $\begin{array}{c}\text { Derechos Humanos } \\
\text { Sociedad }\end{array}$ & 10 \\
\hline $\begin{array}{c}\text { Responsabilidad sobre } \\
\text { productos }\end{array}$ & 9 \\
\hline
\end{tabular}

(C) Intangible Capital, 2013 (www.intangiblecapital.org)

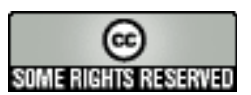

El artículo está con Reconocimiento-NoComercial 3.0 de Creative Commons. Puede copiarlo, distribuirlo y comunicarlo públicamente siempre que cite a su autor y a Intangible Capital. No lo utilice para fines comerciales. La licencia completa se puede consultar en http://creativecommons.org/licenses/by-nc/3.0/es/ 